T. Murai

Nagoya Math. J.

Vol. 61 (1976), 135-159

\title{
ON AREA INTEGRALS AND RADIAL VARIATIONS OF ANALYTIC FUNCTIONS IN THE UNIT DISK
}

\author{
TAKAFUMI MURAI
}

\section{Introduction}

We are concerned with the behaviour of analytic functions near the boundary. Let $T$ and $D$ be the unit circle $|z|=1$ and the unit disk $|z|<1$, respectively. The element of $T$ is denoted by $\theta(0 \leq \theta<2 \pi)$. Let $f(z)=\sum_{n=1}^{\infty} a_{n} z^{n}$ be analytic in $D$. The area integral $A(f, \theta)$ of $f$ at $\theta$ is defined by

$$
A(f, \theta)=\iint_{\Gamma(\theta)}\left|f^{\prime}\left(r e^{i \varphi}\right)\right|^{2} r d r d \varphi,
$$

where $\Gamma(\theta)=\left\{z ;|z|>\frac{1}{2},\left|\arg \left(z-e^{i \theta}\right)\right|<1\right\}$. It represents the area of the image of $\Gamma(\theta)$. We know the following two relations:

(1) The finiteness of $A(f, \theta)$ reflects the existence of $\lim _{r \rightarrow 1} f\left(r e^{i \theta}\right)$.

(2) The infiniteness of $A(f, \theta)$ reflects the totality of $f(\Gamma(\theta))$, that is, $f(\Gamma(\theta))=\{z ;|z|<+\infty\}$.

So it is interesting to know whether $A(f, \theta)$ is finite or not. Our problems are to characterize the finiteness of $A(f, \theta)$ and to study these relations (1) and (2). But it is complicated to examine them for given $f$ and $\theta \in T$. So some authors studied them for a given $f$ occasionally neglecting a small subset of $T$. (cf. Theorem (1.1) in [4] p. 199) The author also took the same line at first. But, in this paper, we shall study them neglecting a class of functions. To define a negligible class of functions, we need a probability space.

Let $(\Omega, \mathfrak{B}, p)$ be a probability space, where $\Omega$ is a space, $\mathfrak{B}$ events and $p$ a probability. Let $X=\left(X_{n}\right)_{n=1}^{\infty}$ be a sequence of independent random variables. Consider a class of analytic functions, so-called a random Taylor series by $X, f_{X}(z)=\sum_{n=1}^{\infty} X_{n} a_{n} z^{n}$. For a random Taylor series $f_{X}$, we shall neglect a class of functions in $f_{X}$ with probability 0 .

Received April 22, 1975. 
From the point of view of random Taylor series, we shall consider the above problems. First, we remark the following fact. The property of the finiteness of $A\left(f_{X}, \theta\right)$ is an event and independent on the values of a finite number of $X_{n} a_{n} z^{n}$. By the zero-one law, we obtain that $A\left(f_{X}, \theta\right)$ $<+\infty$ holds with probability 1 or 0 .

We shall also treat by the same manner the generalized area integrals and the radial variations which are defined in the section 2 .

\section{Definitions}

Let $C$ be the complex plane. The element of $C$ is denoted by $z=$ $r e^{i \varphi}, \zeta, \cdots$ etc. Let $T$ and $D$ be the unit circle and the unit open disk with center zero, respectively. The element of $T$ is denoted by $\theta(0 \leq$ $\theta<2 \pi)$. Let $f(z)=\sum_{n=1}^{\infty} a_{n} z^{n}$ be analytic in $D$.

The area integral $A(f, \theta, \beta)$ of $f$ at $\theta$ is defined by

$$
A(f, \theta, \beta)=\iint_{\Gamma_{\beta}(\theta)}\left|f^{\prime}\left(r e^{i \varphi}\right)\right|^{2} r d r d \varphi
$$

where $\Gamma_{\beta}(\theta)=\left\{z ;|z|>\frac{1}{2},\left|\arg \left(z-e^{i \theta}\right)\right|<\beta\right\}(0<\beta<\pi / 2)$. We denote $A(f, \theta)=A(f, \theta, 1)$. We have two generalizations of $A(f, \theta)$.

The area integral $A_{\alpha}(f, \theta)$ of $f$ of order $\alpha(-1<\alpha<1)$ is defined by

$$
A_{\alpha}(f, \theta)=\int_{0}^{1} r(1-r)^{-\alpha} d r \int_{\theta-(1-r)}^{\theta+(1-r)}\left|f^{\prime}\left(r e^{i \varphi}\right)\right|^{2} d \varphi .
$$

We know that $A_{0}(f, \theta)$ and $A(f, \theta)$ are equivalent in the following sense: There exist $\gamma_{1}, \gamma_{2}\left(0<\gamma_{1}, \gamma_{2}<\pi / 2\right)$ such that $c_{1} A_{0}(f, \theta, \gamma) \leq A(f, \theta) \leq$ $c_{2} A_{0}\left(f, \theta, \gamma_{2}\right)$ for some positive constants $c_{1}, c_{2}$.

The area integral $\tilde{A}_{\alpha}(f, \theta)$ of $f$ of tangency $\alpha\left(0 \leq \alpha \leq \frac{1}{2}\right)$ is defined by

$$
\tilde{A}_{\alpha}(f, \theta)=\int_{0}^{1} r d r \int_{\theta-(1-r)^{1-\alpha}}^{\theta+(1-r)^{1-\alpha}}\left|f^{\prime}\left(r e^{i \varphi}\right)\right|^{2} d \varphi .
$$

The radial variation $V(f, \theta)$ of $f$ is defined by

$$
V(f, \theta)=\int_{0}^{1}\left|f^{\prime}\left(r e^{i \theta}\right)\right| d r
$$

For convenience sake, we write the following notation:

$$
A_{\alpha}^{t}(f, \theta)=\int_{0}^{t} r(1-r)^{-\alpha} d r \int_{\theta-(1-r)}^{\theta+(1-r)}\left|f^{\prime}\left(r e^{i \varphi}\right)\right|^{2} d \varphi \quad(0<t<1)
$$




$$
c_{\alpha}(n, m ; t)=n m \int_{0}^{t} r^{n+m-1}(1-r)^{-\alpha} \int_{-1+r}^{1-r} \cos (n-m) \varphi d \varphi
$$

where $n, m$ are integers. We denote $c_{\alpha}(n, m)=c_{\alpha}(n, m ; 1)$. Let $f(z)$ $=\sum_{n=1}^{\infty} a_{n} z^{n}$ be analytic in $D$. We have

$$
\begin{aligned}
A_{\alpha}^{t}(f, \theta) & =\int_{0}^{t} r(1-r)^{-\alpha} d r \int_{\theta-(1-r)}^{\theta+(1-r)}\left|\sum_{n=1}^{\infty} n a_{n} r^{n-1} e^{i(n-1) \varphi}\right|^{2} d \varphi \\
& =\sum_{n=1}^{\infty} \sum_{m=1}^{\infty} c_{\alpha}(n, m ; t) a_{n} e^{i n \theta} \overline{a_{m} e^{i m \theta}} .
\end{aligned}
$$

In this paper, we use the following notation: If the inequality $0 \leq f(z) \leq c g(z)$ holds for some positive constant $c$, we denote $f(z) \lesssim g(z)$. If the inequality $c_{1} f(z) \leq g(z) \leq c_{2} f(z)$ holds for some positive constants $c_{1}, c_{2}$, we denote $f(z) \approx g(z)$.

Next, we define the probability space $(\Omega, \mathfrak{B}, p)$ which is fixed throughout this paper. Let $I$ be the interval $[0,1)$ and let $\left(I, \mathfrak{B}_{I}, p_{I}\right)$ be the usual probability space. Set $\Omega=\prod_{n=1}^{\infty} I_{n}$, where $I_{n}=I$ for all $n$. Then the product space $(\Omega, \mathfrak{B}, p)$ is usually defined. The element of $\Omega$ is denoted by $\omega$. The expectation is denoted by $\mathscr{E}[\cdot]$. We consider a sequence $X=\left(X_{n}\right)_{n=1}^{\infty}$ of independent random variables which satisfies the following conditions :

(i) $X_{n}$ is real-valued.

(ii) $X_{n}$ is a random variable on $I_{n}$.

(iii) $X_{n}$ is symmetric, that is, $p\left(X_{n}>c\right)=p\left(-X_{n}>c\right)$ for all $c \geq 0$.

(iv) $\sup _{n}\left[X_{n}^{2}\right]<+\infty$.

( v ) $\sup _{n} \mathscr{E}\left[X_{n}^{4}\right] \mathscr{E}\left[X_{n}^{2}\right]^{-2}<+\infty$.

As a technique, we shall use a Rademacher series which is defined as follows. Let $J$ be two points $\{-1,1\}$. Set $\widetilde{\Omega}=\prod_{n=1}^{\infty} J_{n}$, where $J_{n}$ $=J$ for all $n$. Then the usual probability space $(\widetilde{\Omega}, \tilde{\mathfrak{B}}, \tilde{p})$ is defined. The element of $\tilde{\Omega}$ is denoted by $x$. A Rademacher series $\varepsilon=\left(\varepsilon_{n}\right)_{n=1}^{\infty}$ is defined by

(a) $\varepsilon_{n}$ is a random variable on $J_{n}$

(b) $\varepsilon_{n}(-1)=-1, \varepsilon_{n}(1)=1$.

Then $\varepsilon=\left(\varepsilon_{n}\right)_{n=1}^{\infty}$ is a sequence of independent random variables with $\widetilde{p}\left(\varepsilon_{n}=1\right)=\tilde{p}\left(\varepsilon_{n}=-1\right)=\frac{1}{2}(n=1,2, \cdots)$.

If some property $\boldsymbol{P}_{1}$ on $\Omega$ hold with probability 1 , we say that $\boldsymbol{P}_{1}$ 
holds almost surely (a.s.). If some property $\boldsymbol{P}_{2}$ on $\boldsymbol{T}$ holds with Lebesgue measure $2 \pi$, we say that $\boldsymbol{P}_{2}$ holds almost everywhere (a.e.).

\section{Immediate consequences and constructions of examples}

We first show the following

Proposition 1. Let $|\alpha|<1$ and let $f_{X}(z)=\sum_{n=1}^{\infty} X_{n} a_{n} z^{n}$ be a random Taylor series defined by $X=\left(X_{n}\right)_{n=1}^{\infty}$. Then $A_{\alpha}\left(f_{X}, 0\right)<+\infty$ a.s. if and only if $\sum_{n=1}^{\infty} \mathscr{E}\left[\left|X_{n}\right|^{2}\right] n^{\alpha}\left|a_{n}\right|^{2}<+\infty \cdots(*)_{\alpha}$.

For the proof, we prepare the following

LEMMA 1 ([1] p. 6). Let $Y$ be a positive random variable. Then for $0<\lambda<1$, we have

$$
p(Y \geq \lambda \mathscr{E}[Y]) \geq(1-\lambda)^{2} \mathscr{E}[Y]^{2} \mathscr{E}\left[Y^{2}\right]^{-1}
$$

Proof of Proposition 1. First we remark $\int_{0}^{1} r^{2 n-1}(1-r)^{1-\alpha} d r \approx n^{\alpha-2}$. Assume that $(*)_{\alpha}$ holds. From the hypothesis (v), we have, with some constant $c, \mathscr{E}\left[X_{n}^{4}\right] \leq c \mathscr{E}\left[X_{n}^{2}\right]^{2}$. Since

$$
A_{\alpha}^{t}\left(f_{X}, 0\right)=\sum_{n=1}^{\infty} \sum_{m=1}^{\infty} X_{n} X_{m} c_{\alpha}(n, m ; t) a_{n} \bar{a}_{m},
$$

it follows from (iii) that

$$
\begin{aligned}
\mathscr{E}\left[A_{\alpha}^{t}\left(f_{X}, 0\right)\right] & =\sum_{n=1}^{\infty} \sum_{m=1}^{\infty} \mathscr{E}\left[X_{n} X_{m}\right] c_{\alpha}(n, m ; t) a_{n} \bar{a}_{m} \\
& =\sum_{n=1}^{\infty} \mathscr{E}\left[X_{n}^{2}\right] c_{\alpha}(n, n ; t)\left|a_{n}\right|^{2} .
\end{aligned}
$$

Letting $t$ tend to 1 , we have

$$
\mathscr{E}\left[A_{\alpha}\left(f_{X}, 0\right)\right]=\sum_{n=1}^{\infty} \mathscr{E}\left[X_{n}^{2}\right] c_{\alpha}(n, n)\left|a_{n}\right|^{2} \approx \sum_{n=1}^{\infty} \mathscr{E}\left[X_{n}^{2}\right] n^{\alpha}\left|a_{n}\right|^{2}<+\infty .
$$

Hence $A_{a}\left(f_{X}, 0\right)<+\infty$ a.s..

Conversely, assume that $A_{\alpha}\left(f_{X}, 0\right)<+\infty$ hold a.s.. We shall apply the above lemma to the random variable $A_{\alpha}^{t}\left(f_{X}, 0\right)$. We have

$$
\mathscr{E}\left[A_{\alpha}^{t}\left(f_{x}, 0\right)\right]^{2}=\left(\sum_{n=1}^{\infty} \mathscr{E}\left[X_{n}^{2}\right] c_{\alpha}(n, n ; t)\left|a_{n}\right|^{2}\right)^{2}
$$

and

$$
\mathscr{E}\left[A_{\alpha}^{t}\left(f_{X}, 0\right)^{2}\right]=\mathscr{E}\left[\left(\sum_{n, m} X_{n} X_{m} c_{\alpha}(n, m ; t) a_{n} \bar{a}_{m}\right)^{2}\right]
$$




$$
\begin{aligned}
= & \mathscr{E}\left[\sum_{n_{1} m_{1} n_{2} m_{2}} X_{n_{1}} X_{m_{1}} X_{n_{2}} X_{m_{2}} c_{\alpha}\left(n_{1}, m_{1} ; t\right) c_{\alpha}\left(n_{2}, m_{2} ; t\right) a_{n_{1}} \bar{a}_{m_{1}} a_{n_{2}} \bar{a}_{m_{2}}\right] \\
= & \sum_{n_{1} m_{1} n_{2} m_{2}} \mathscr{E}\left[X_{n_{1}} X_{m_{1}} X_{n_{2}} X_{m_{2}}\right] c_{\alpha}\left(n_{1}, m_{1} ; t\right) c_{\alpha}\left(n_{2}, m_{2} ; t\right) a_{n_{1}} \bar{a}_{m_{1}} a_{n_{2}} \bar{a}_{m_{2}} \\
\leq & \sum_{n, m} \mathscr{E}\left[X_{n}^{2} X_{m}^{2}\right] c_{\alpha}(n, n ; t) c_{\alpha}(m, m ; t)\left|a_{n}\right|^{2}\left|a_{m}\right|^{2} \\
& +\sum_{n, m} \mathscr{E}\left[X_{n}^{2} X_{m}^{2}\right] c_{\alpha}(n, m ; t)^{2}\left|a_{n}\right|^{2}\left|a_{m}\right|^{2} .
\end{aligned}
$$

Since we have

$$
\left.\mathscr{E}\left[X_{n}^{2} X_{m}^{2}\right] \leq \sqrt{\mathscr{E}\left[X_{n}^{4}\right]} \sqrt{\mathscr{E}\left[X_{m}^{4}\right.}\right] \leq c \mathscr{E}\left[X_{n}^{2}\right] \mathscr{E}\left[X_{m}^{2}\right]
$$

and

$$
c_{\alpha}(n, m ; t) \leq n m \int_{0}^{1} r^{m+m-1}(1-r)^{-\alpha} d r \int_{-1+r}^{1-r} d \varphi \leq \sqrt{c_{\alpha}(n, n ; t)} \sqrt{c_{\alpha}(m, m ; t)}
$$

we obtain

$$
\mathscr{E}\left[A_{\alpha}^{t}\left(f_{X}, 0\right)^{2}\right] \leq 2 c\left(\sum_{n=1}^{\infty} \mathscr{E}\left[X_{n}^{2}\right] c_{\alpha}(n, n ; t)\left|a_{n}\right|^{2}\right)^{2}
$$

Therefore

$$
\mathscr{E}\left[A_{\alpha}^{t}\left(f_{X}, 0\right)\right]^{2} \mathscr{E}\left[A_{\alpha}^{t}\left(f_{X}, 0\right)^{2}\right]^{-2} \geq \frac{1}{2 c}
$$

By Lemma 1, we have

$$
p\left(A_{\alpha}^{t}\left(f_{X}, 0\right) \geq \frac{1}{2} \mathscr{E}\left[A_{\alpha}^{t}\left(f_{X}, 0\right)\right]\right) \geq\left(1-\left(\frac{1}{2}\right)\right) \frac{1}{2 c}(=\eta)>0 .
$$

Choose a sequence $\left(t_{n}\right)_{n=1}^{\infty}$ such that $0<t_{n}<1$ and $t_{n} \uparrow 1$. Set

$$
E_{n}=\left\{A_{\alpha}^{t_{n}}\left(f_{X}, 0\right) \geq \frac{1}{2} \mathscr{E}\left[A_{\alpha}^{t_{n}}\left(f_{X}, 0\right)\right]\right\}
$$

Since $p\left(E_{n}\right) \geq \eta$ for all $n$, we have $p\left(\lim \sup _{n \rightarrow \infty} E_{n}\right) \geq \eta$. By the assumption, there exists $\omega \in \lim \sup _{n \rightarrow \infty} E_{n}$ such that $A_{\alpha}\left(f_{X(\omega)}, 0\right)<+\infty$. Then we have

$$
\begin{gathered}
\sum_{n=1}^{\infty} \mathscr{E}\left[X_{n}^{2}\right] n^{\alpha}\left|a_{n}\right|^{2} \approx \mathscr{E}\left[A_{\alpha}\left(f_{X}, 0\right)\right]=\lim _{n \rightarrow \infty} \mathscr{E}\left[A_{\alpha}^{t_{n}}\left(f_{X}, 0\right)\right] \\
\leq \lim _{n \rightarrow \infty} A_{\alpha}^{t_{n}}\left(f_{X(\omega)}, 0\right)=A_{\alpha}\left(f_{X(\omega)}, 0\right)<+\infty
\end{gathered}
$$

This completes the proof. 
Corollary 1. Let $|\alpha|<1$ and $f_{X}$ be the same as in Proposition 1. Then $A_{\alpha}\left(f_{X}, \theta\right)<+\infty$ a.e. holds a.s. if and only if $(*)_{\alpha}$ holds.

Proof. Consider the product space $\left(\Omega \times T, \mathfrak{B} \times \mathfrak{B}_{T}, p \times d \theta\right)$. We denote by $\tilde{\mathscr{E}}[\cdot]$ the expectation. Define a sequence $Y=\left(Y_{n}\right)_{n=1}^{\infty}$ of random variables on $\Omega \times T$ by $Y_{n}(\omega, \theta)=\dot{X}_{n}(\omega) e^{i n \theta}$. Then we have

$$
\sup _{n} \widetilde{\mathscr{E}}\left[\left|Y_{n}\right|^{4}\right] \widetilde{\mathscr{E}}\left[\left|Y_{n}\right|^{2}\right]^{-2}=\sup _{n} \mathscr{E}\left[X_{n}^{4}\right] \mathscr{E}\left[X_{n}^{2}\right]^{-2}<+\infty
$$

and

$$
\widetilde{E}\left[Y_{n_{1}} Y_{m_{1}} \bar{Y}_{n_{2}} \bar{Y}_{m_{2}}\right]=2 \pi \mathscr{E}\left[X_{n_{1}} X_{m_{1}} X_{n_{2}} X_{m_{2}}\right] \delta_{n_{1}+m_{1}, n_{2}+m_{2}},
$$

where $\delta_{n, m}$ means Kronecker's. By the same method as in Proposition 1, we know that $A_{\alpha}\left(f_{Y}, 0\right)<+\infty$ a.s. $(p \times d \theta)$ if and only if $(*)_{\alpha}$ holds. Since $A_{\alpha}\left(f_{Y(\omega, \theta)}, 0\right)=A_{\alpha}\left(f_{X(\omega)}, \theta\right)$, we know that $A_{\alpha}\left(f_{X}, \theta\right)<+\infty$ a.e. holds a.s. if and only if $(*)_{\alpha}$ holds, this completes the proof.

Proposition 1'. Let $f_{X}(z)=\sum_{n=1}^{\infty} X_{n} a_{n} z^{n}$ be a random Taylor series. Set $s_{j}=\left(\sum_{2^{j} \leq n<2^{j+1}} \mathscr{E}\left[X_{n}^{2}\right]\left|a_{n}\right|^{2}\right)^{1 / 2}$. If $\sum_{j=0}^{\infty} s_{j}<+\infty$, then $V\left(f_{X}, 0\right)<+\infty$ a.s..

Proof. We have

$$
V\left(f_{X}, 0\right)=\int_{0}^{1}\left|f_{X}^{\prime}(r)\right| d r \leq \sum_{j=0}^{\infty} \int_{0}^{1}\left|\sum_{2^{j} \leq n<2^{j+1}} n X_{n} a_{n} r^{n-1}\right| d r .
$$

Since we have

$$
\begin{aligned}
\mathscr{E}\left[\sum_{2^{j} \leq n<2^{j+1}} X_{n} n a_{n} r^{n-1} \mid\right] & \leq \mathscr{E}\left[\sum_{2^{j} \leq n, m<2^{j+1}} X_{n} X_{m} n m a_{n} \bar{a}_{m} r^{n+m-2}\right]^{1 / 2} \\
& \leq\left(\sum_{2 j \leq n<2^{j+1}} \mathscr{E}\left[X_{n}^{2}\right] n^{2}\left|a_{n}\right|^{2} r^{2 n-2}\right)^{1 / 2} \leq 2^{j+1} r^{2 j-1} s_{j},
\end{aligned}
$$

we obtain

$$
\mathscr{E}\left[V\left(f_{X}, 0\right)\right] \leq \sum_{j=0}^{\infty} s_{j} 2^{j+1} \int_{0}^{1} r^{2^{j-1}} d r \approx \sum_{j=0}^{\infty} s_{j}<+\infty
$$

Therefore we have $V\left(f_{X}, 0\right)<+\infty$ a.s.. This completes the proof.

CoRollary 1'. If $\sum_{j=0}^{\infty} s_{j}<+\infty$, then $V\left(f_{X}, \theta\right)<+\infty$ a.e. holds a.s..

This is easily proved by the same method as in Proposition $1^{\prime}$. Hence we omit the proof.

Remark 1. The similar assertion as in Proposition 1 for $\tilde{A}_{\alpha}(0<\alpha$ 
$\left.\leq \frac{1}{2}\right)$ holds. Now, choose a sequence $\left(a_{n}\right)_{n=1}^{\infty}$ such that $\sum_{n=1}^{\infty} n^{\alpha}\left|a_{n}\right|^{2}<+\infty$ and $\sum_{n=1}^{\infty} n^{\beta}\left|a_{n}\right|^{2}=+\infty \quad\left(0 \leq \alpha<\beta \leq \frac{1}{2}\right)$. Consider a random Taylor series $f_{\mathrm{c}}(z)=\sum_{n=1}^{\infty} \varepsilon_{n} a_{n} z^{n}$. Then we have almost surely $A_{\alpha}\left(f_{\mathrm{e}}, \theta\right)<+\infty$, $A_{\beta}\left(f_{\mathrm{e}}, \theta\right)=+\infty, \tilde{A}_{\alpha}\left(f_{\mathrm{e}}, \theta\right)<+\infty$ and $\tilde{A}_{\alpha}\left(f_{\mathrm{e}}, \theta\right)=+\infty$ a.e..

Proposition 2. Let $X=\left(X_{n}\right)_{n=1}^{\infty}$ be a sequence of independent realvalued normal Gaussian variables (i.e. $p\left(X_{n}<t\right)=1 / \sqrt{2 \pi} \int_{-\infty}^{t} e^{-s^{2} / 2} d s$ ) and let $f_{X}(z)=\sum_{n=1}^{\infty} X_{n} a_{n} z^{n}$ be a random Taylor series. Then $V\left(f_{X}, 0\right)<+\infty$ a.s. if and only if $\int_{0}^{1} \sqrt{\sum_{n=1}^{\infty} n^{2}\left|a_{n}\right|^{2} r^{2 n-2}} d r<+\infty$.

Proof. We can assume that $a_{n}$ 's are real. We have

$$
\mathscr{E}\left[V\left(f_{X}, 0\right)\right]=\int_{0}^{1} \mathscr{E}\left[\left|\sum_{n=1}^{\infty} X_{n} n a_{n} r^{n-1}\right|\right] d r=\sqrt{\frac{2}{\pi}} \int_{0}^{1} \sqrt{\sum_{n=1}^{\infty} n^{2}\left|a_{n}\right|^{2} r^{2 n-2}} d r .
$$

Hence 'if' part holds. Set $V^{t}\left(f_{X}, 0\right)=\int_{0}^{t}\left|f_{X}^{\prime}\right| d r$. We shall show that $\mathscr{E}\left[V^{t}\left(f_{X}, 0\right)^{2}\right] \mathscr{E}\left[V^{t}\left(f_{X}, 0\right)\right]^{-2} \leq 4$ for all $0<t<1$. We have

$$
\mathscr{E}\left[V^{t}\left(f_{X}, 0\right)\right]^{2}=\frac{2}{\pi}\left(\int_{0}^{t} \sqrt{\sum_{n=1}^{\infty} n^{2}\left|a_{n}\right|^{2} \cdot r^{2 n-2}} d r\right)^{2}
$$

and

$\mathscr{E}\left[V^{t}\left(f_{X}, 0\right)^{2}\right]$

$$
\begin{aligned}
& =\int_{0}^{t} \int_{0}^{t} \mathscr{E}\left[\left|\sum_{n=1}^{\infty} X_{n} n a_{n} r^{n-1}\right|\left|\sum_{n=1}^{\infty} X_{n} n a_{n} s^{n-1}\right|\right] d r d s \\
& =\int_{0}^{t} \int_{0}^{t} d r d s \frac{1}{\sqrt{A B-C^{2}}} \int_{-\infty}^{\infty} \int_{-\infty}^{\infty}|x||y| \exp \left(-\pi \frac{B x^{2}+A y^{2}-2 C x y}{A B-C^{2}}\right) d x d y,
\end{aligned}
$$

where

$$
A=\mathscr{E}\left[\left|\sum_{n=1}^{\infty} X_{n} n a_{n} r^{n-1}\right|^{2}\right]=\sum_{n=1}^{\infty} n^{2}\left|a_{n}\right|^{2} r^{2 n-2}, \quad B=\sum_{n=1}^{\infty} n^{2}\left|a_{n}\right|^{2} s^{2 n-2}
$$

and

$$
C=\mathscr{E}\left[\sum_{n=1}^{\infty} X_{n} n a_{n} r^{n-1} \sum_{n=1}^{\infty} X_{n} n a_{n} s^{n-1}\right]=\sum_{n=1}^{\infty} n^{2}\left|a_{n}\right|^{2} r^{n-1} s^{n-1}
$$

Since

$$
\begin{aligned}
& \frac{1}{\sqrt{\overline{A B-C^{2}}}} \int_{-\infty}^{\infty} \int_{-\infty}^{\infty}|x||y| \exp \left(-\pi \frac{B x^{2}+A y^{2}-2 C x y}{A B-C^{2}}\right) d x d y \\
& \leq 4 \sqrt{A B-C^{2}} \leq 4 \sqrt{A B},
\end{aligned}
$$


we have

$$
\mathscr{E}\left[V^{t}\left(f_{X}, 0\right)^{2}\right] \leq 4\left(\int_{0}^{t} \sqrt{\sum_{n=1}^{\infty} n^{2}\left|a_{n}\right|^{2} r^{2 n-2}} d r\right)^{2} .
$$

Therefore $\mathscr{E}\left[V^{t}\left(f_{X}, 0\right)^{2}\right] \mathscr{E}\left[V^{t}\left(f_{X}, 0\right)\right]^{-2} \leq 4$. Hence the rest of the proof follows in the same manner as in Proposition 1. This completes the proof.

To discuss the sure properties, we consider lacunary series. Let $\left(\ell_{\alpha}(k)\right)_{k=0}^{\infty}(0<\alpha<1)$ be a sequence of positive integers such that (1 $\alpha) \ell_{\alpha}(k+1) \geq 2 \ell_{\alpha}(k)$. We denote by $N_{\alpha}(k)=2^{\ell \alpha(k)}$ and $N(k)=2^{2 k}$ throughout this paper.

Proposition 5. Let $0<\alpha<1$ and let $\left(a_{n}\right)_{n=1}^{\infty}$ be a bounded sequence such that $a_{n}=0$ for $n \neq N_{\alpha}(k)(k=0,1, \cdots)$. Set $f(z)=\sum_{n=1}^{\infty} a_{n} z^{n}$. Then $A_{\alpha}(f, \theta)<+\infty$ for all $\theta$ or $A_{\alpha}(f, \theta)=+\infty$ for all $\theta$ according to $\sum_{n=1}^{\infty} n^{\alpha}\left|a_{n}\right|^{2}<+\infty$ or $=+\infty$.

Proof. We can assume $\left|a_{n}\right| \leq 1$ for all $n$. We have

$$
\begin{aligned}
A_{\alpha}^{t}(f, \theta)= & \sum_{n=1}^{\infty} \sum_{m=1}^{\infty} c_{\alpha}(n, m ; t) a_{n} e^{i n \theta} \overline{a_{m} e^{i m \theta}} \\
= & \sum_{k=0}^{\infty} c_{\alpha}\left(N_{\alpha}(k), N_{\alpha}(k) ; t\right)\left|a_{N_{\alpha}(k)}\right|^{2} \\
& +2 \operatorname{Re}\left(\sum_{k=1}^{\infty} \sum_{k^{\prime}=0}^{k-1} c_{\alpha}\left(N_{\alpha}(k), N_{\alpha}\left(k^{\prime}\right) ; t\right) a_{N_{\alpha}(k)} \bar{a}_{N_{\alpha}\left(k^{\prime}\right)} e^{i\left(N_{\alpha}(k)-N_{\alpha}\left(k^{\prime}\right)\right) \theta}\right) .
\end{aligned}
$$

We have the following estimation:

$$
\begin{aligned}
\mid(\text { The second term }) \mid & \lesssim \sum_{k=1}^{\infty} \sum_{k^{\prime}=0}^{k-1} N_{\alpha}(k) N_{\alpha}\left(k^{\prime}\right)\left(N_{\alpha}(k)+N_{\alpha}\left(k^{\prime}\right)\right)^{\alpha-2} \\
& \leq \sum_{k=1}^{\infty} N_{\alpha}(k)^{\alpha-1} \cdot k \cdot N_{\alpha}(k-1)<+\infty .
\end{aligned}
$$

Letting $t$ tend to 1 , we have $A_{\alpha}(f, \theta) \approx \sum_{n=1}^{\infty} n^{\alpha}\left|a_{n}\right|^{2}+0(1)$. This completes the proof.

Proposition 5'. Let $0<\alpha<1$ and let $\left(a_{n}\right)_{n=1}^{\infty}$ be an absolutely convergent sequence such that $a_{n}=0$ for $n \neq N(k)(k=0,1, \cdots)$. Set $f(z)$ $=\sum_{n=1}^{\infty} a_{n} z^{n}$. Then $A_{\alpha}(f, \theta)<+\infty$ for all $\theta$ or $A_{\alpha}(f, \theta)=+\infty$ for all $\theta$ according to $\sum_{n=1}^{\infty} n^{\alpha}\left|a_{n}\right|^{2}<+\infty$ or $=+\infty$.

By using the following estimation, we have $A_{\alpha}(f, \theta) \approx \sum_{n=1}^{\infty} n^{\alpha}\left|a_{n}\right|^{2}$ $+0(1)$. 


$$
\begin{aligned}
& \left|\sum_{k=1}^{\infty} \sum_{k^{\prime}=0}^{\infty} c_{\alpha}\left(N(k), N\left(k^{\prime}\right) ; t\right) a_{N(k)} \bar{a}_{N\left(k^{\prime}\right)} e^{i\left(N(k)-N\left(k^{\prime}\right)\right) \theta}\right| \\
& \quad \lesssim \sum_{k=1}^{\infty}\left|a_{N(k)}\right| \sum_{k^{\prime}=0}^{k-1}\left|a_{N\left(k^{\prime}\right)}\right| N(k) N\left(k^{\prime}\right)\left(N(k)+N\left(k^{\prime}\right)\right)^{\alpha-2} \\
& \quad \leq\left(\sum_{k=1}^{\infty}\left|a_{N(k)}\right|\right)^{2}<+\infty .
\end{aligned}
$$

COROLlaRY 2. There exists an absolutely convergent Taylor series $f(z)=\sum_{n=1}^{\infty} a_{n} z^{n}$ such that $A_{\alpha}(f, \theta)=+\infty$ for all $\theta$ and all $0<\alpha<1$.

Proof. Let $\left(a_{n}\right)_{n=1}^{\infty}$ be a sequence such that $a_{N(k)}=(k+1)^{-2}(k=0,1, \cdots)$ $a_{n}=0 n \neq N(k)$. Then $\sum_{n=0}^{\infty} n^{\alpha}\left|a_{n}\right|^{2}=+\infty$ for all $0<\alpha<1$. By Proposition $2^{\prime}, A_{\alpha}(f, \theta)=+\infty$ for all $\theta$ and $0<\alpha<1$. This completes the proof.

Remark 2. By [2], $\theta \in \boldsymbol{T}$ is called a Lusin point of $f$ if $\tilde{A}_{1 / 2}(f, \theta, t)$ $=\iint_{\left|z-t e^{i \theta}\right|<1-t}\left|f^{\prime}(z)\right|^{2} r d r d \varphi$ diverges for all $0<t<1$. We know that there exists a bounded function such that every point $\theta \in T$ is a Lusin point of it ([2]). Let $f$ be the function in Corollary 2. Then every point $\theta \in \boldsymbol{T}$ is a Lusin point of $f$. We shall show it. We have $\tilde{A}_{1 / 2}(f, \theta)$ $=+\infty$ for each $\theta$. We can assume $t>\frac{1}{2}$. If we choose suitable constants $\beta_{t}, \gamma_{t, f}$, we have, for each $\theta$,

$$
\begin{aligned}
\tilde{A}_{1 / 2}(f, \theta, t)= & \iint_{\substack{\mid z-t t^{i \theta \theta \mid<1-t} \\
r<t}}\left|f^{\prime}(z)\right|^{2} r d r d \varphi \\
& +\int_{t}^{1} r d r \int_{|\varphi-\theta|<\operatorname{arc} \cos \left(2 t-1+r^{2}\right)(2 r t)-1}\left|f^{\prime}\right|^{2} d \varphi \\
\geq & \int_{t}^{1} r d r \int_{|\varphi-\theta|<\beta_{t} \sqrt{1-r}}\left|f^{\prime}\right|^{2} d \varphi \approx A_{1 / 2}(f, \theta)+\gamma_{t, f}=+\infty .
\end{aligned}
$$

Therefore $\tilde{A}_{1 / 2}(f, \theta, t)=+\infty$ for all $\theta \in T$ and all $0<t<1$. But there exists $g(z)=\sum_{n=1}^{\infty} b_{n} z^{n}$ such that each $\theta \in \boldsymbol{T}$ is not a Lusin point of $g$ and $A_{\alpha}(g, \theta)=+\infty$ for all $\theta$ and all $\alpha>\frac{1}{2}$. For example, put $b_{N(k)}=$ $k^{-1 / 2} N(k)^{-1 / 4}(k=1,2, \cdots)$ and $b_{n}=0$ for $n \neq N(k)$.

EXAMPLE. There exists an analytic function $f$ such that $V(f, \theta)$ $=+\infty$ and $A_{0}(f, \theta)<+\infty$ for all $\theta$.

Put $b_{N(k)}=k^{-1 / 2} N(k)^{1 / 2}(k=1,2, \cdots)$ and $b_{n}=0$ for $n \neq N(k) \quad(k=$ $1,2, \cdots)$. Consider $f(z)=\int_{0}^{z}\left(\sum_{n=0}^{\infty} b_{n} \zeta^{n}\right)^{2} d \zeta$. We show that $f$ satisfies the required conditions. We have 


$$
\begin{aligned}
V(f, \theta)= & \int_{0}^{1}\left|\sum_{n=1}^{\infty} b_{n} r^{n} e^{i n \theta}\right|^{2} d r \\
= & \sum_{k=1}^{\infty} \sum_{k^{\prime}=1}^{\infty} b_{N(k)} b_{N\left(k^{\prime}\right)}\left(N(k)+N\left(k^{\prime}\right)+1\right)^{-1} e^{i\left(N(k)-N\left(k^{\prime}\right)\right) \theta} \\
= & \sum_{k=1}^{\infty} b_{N(k)}^{2}(2 N(k)+1)^{-1} \\
& +\sum_{k=1}^{\infty} \sum_{k^{\prime} \neq k}^{\infty} b_{N(k)} b_{N\left(k^{\prime}\right)}\left(N(k)+N\left(k^{\prime}\right)+1\right)^{-1} e^{i\left(N(k)-N\left(k^{\prime}\right)\right)} \theta .
\end{aligned}
$$

We have the following estimation:

(The first term) $\approx \sum_{k=1}^{\infty} k^{-1}=+\infty$

$\mid$ (The second term) $\mid \lesssim \sum_{k=1}^{\infty} b_{N(k)} N(k)^{-1} \sum_{k^{\prime}=1}^{k-1} b_{N\left(k^{\prime}\right)} \leq \sum_{k=2}^{\infty} N(k-2)^{-1}<+\infty$.

Therefore we have $V(f, \theta)=+\infty$ for all $\theta$. On the other hand, we have

$$
\begin{aligned}
& A_{0}(f, \theta)= \int_{0}^{1} d r \int_{\theta-(1-r)}^{\theta+(1-r)}\left|\sum_{n=1}^{\infty} b_{n} r^{n} e^{i n \varphi}\right|^{4} d \varphi \\
&= \int_{0}^{1} d r \int_{\theta-(1-r)}^{\theta+(1-r)} \sum_{k_{1}, k_{2}, k_{3}, k_{4}=1}^{\infty} b_{N\left(k_{1}\right)} b_{N\left(k_{2}\right)} b_{N\left(k_{3}\right)} b_{N\left(k_{4}\right)} r^{N\left(k_{1}\right)+N\left(k_{3}\right)+N\left(k_{3}\right)+N\left(k_{4}\right)} \\
& \quad \times e^{i\left(N\left(k_{1}\right)+N\left(k_{2}\right)-N\left(k_{3}\right)-N\left(k_{4}\right)\right) \varphi} d \varphi \\
& \approx \\
& k_{k_{1}, k_{2}, k_{3}, k_{k}=1}^{\infty} b_{N\left(k_{1}\right)} b_{N\left(k_{2}\right)} b_{N\left(k_{3}\right)} b_{N\left(k_{4}\right)}\left(N\left(k_{1}\right)+N\left(k_{2}\right)+N\left(k_{3}\right)+N\left(k_{4}\right)\right)^{-2} \\
& \lesssim \sum_{k=1}^{\infty} b_{N(k)}^{4} N(k)^{-2}+\sum_{k=1}^{\infty} b_{N(k)}^{3} N(k)^{-2} \sum_{k^{\prime}=1}^{k-1} b_{N\left(k^{\prime}\right)}+\sum_{k=1}^{\infty} b_{N(k)}^{2} N(k)^{-2}\left(\sum_{k^{\prime}=1}^{k-1} b_{N\left(k^{\prime}\right)}\right)^{2} \\
&+\sum_{k=1}^{\infty} b_{N(k)} N(k)^{-2}\left(\sum_{k^{\prime}=1}^{k-1} b_{N\left(k^{\prime}\right)}\right)^{3} \\
& \lesssim \sum_{k=1}^{\infty} k^{-2}+\sum_{k=2}^{\infty} k^{-1} N(k-2)^{-1}+\sum_{k=1}^{\infty} N(k-1)^{-1}+\sum_{k=2}^{\infty} k^{2} N(k-2)^{-2} \\
&+O(1)<+\infty .
\end{aligned}
$$

Therefore we have $A_{0}(f, \theta)<+\infty$ for all $\theta$.

\section{Almost sure property for all $\theta$}

THEOREM 1. Let $|\alpha|<1$ and $f_{X}(z)=\sum_{n=1}^{\infty} X_{n} a_{n} z^{n}$ be a random Taylor series. Set $s_{j}=\sqrt{\sum_{2^{j} \leq n<2^{j+1}}} \mathscr{E}\left[X_{n}^{2}\right] n^{\alpha}\left|a_{n}\right|^{2}(j=0,1, \cdots)$. If $s_{j} \downarrow 0$ and $\sum_{j=0}^{\infty} s_{j}<+\infty$, then $A_{\alpha}\left(f_{X}, \theta\right)$ is bounded ((as a function of $\theta$ ) a.s..

We denote by $\|P\|_{\infty}=\sup _{\theta \in \boldsymbol{T}}|P(\theta)|$ for a continuous function $P$ on $\boldsymbol{T}$. We use the following 
LEMMA 2. ([1] p. 55) Let $\left(P_{n}\right)_{n=1}^{\ell}$ be a sequence of trigonometric polynomials of degree $\leq N$. Set $P_{s}=\sum_{n=1}^{\ell} \varepsilon_{n} P_{n}$. Then we have, with positive constants $c_{1}, c_{2}$,

$$
\tilde{p}\left(\left(\left\|P_{\varepsilon}\right\|_{\infty} \geq c_{1}(\log N)^{1 / 2}\left(\sum_{n=1}^{\ell}\left\|P_{n}\right\|_{\infty}^{2}\right)^{1 / 2}\right) \leq c_{2} N^{-2} .\right.
$$

Proof of Theorem 1. First we consider the case of a Rademacher series. We denote $R_{s k}(z)=\sum_{N(k) \leq n<N(k+1)} \varepsilon_{n} a_{n} z^{n}(k=0,1, \ldots)$. We have

$$
\sqrt{A_{\alpha}\left(f_{s}, \theta\right)} \leq \sqrt{A_{\alpha}\left(a_{1} z, \theta\right)}+\sum_{k=0}^{\infty} \sqrt{A_{\alpha}\left(R_{\epsilon k}, \theta\right)} .
$$

We show

$$
\begin{aligned}
& \tilde{p}\left(\sqrt{\left\|A_{\alpha}\left(R_{s k}, \cdot\right)\right\|_{\infty}} \geq c_{1}(\log N(k+1))^{1 / 2}\left(\sum_{N(k) \leq n<N(k+1)} c_{\alpha}(n, n)\left|a_{n}\right|^{2}\right)^{1 / 2}\right) \\
& \leq c_{2} N(k+1)^{-1} .
\end{aligned}
$$

Set $\quad \ell(k)=N(k+1)-N(k), \quad \tilde{\varepsilon}_{\mu}=\varepsilon_{N(k)-1+\mu}, \quad b_{\mu}=a_{N(k)-1+\mu} \quad$ and $\quad b_{\mu}(\theta)=$ $a_{N(k)-1+\mu} e^{i(N(k)-1+\mu) \theta} \quad(\mu=1, \cdots, \ell(k))$. We denote by $b_{s}(\theta)=\left(\tilde{\varepsilon}_{1} b_{1}(\theta), \cdots\right.$, $\left.\tilde{\varepsilon}_{\ell(k)} b_{\ell(k)}(\theta)\right)$ and

$$
\begin{aligned}
C & =\left(c_{\mu \nu}\right)_{\mu, \nu=1, \cdots, \ell(k)} \\
& =\left(\begin{array}{c}
c_{\alpha}(N(k), N(k)), \cdots c_{\alpha}(N(k), N(k+1)-1) \\
\ddots \\
c_{\alpha}(N(k+1)-1, N(k)), \cdots c_{\alpha}(N(k+1)-1, N(k+1)-1)
\end{array}\right) .
\end{aligned}
$$

Since $C$ is positive definite, there exists a unitary matrix $U=\left(u_{\mu \nu}\right)_{\mu \nu=1}$, $\cdots, \ell(k)$ such that $U^{*} C U=\left(\begin{array}{lll}\lambda_{1} & & 0 \\ & \ddots & \\ 0 & & \lambda_{\ell(k)}\end{array}\right)$, where $\left\{\lambda_{\mu}\right\}_{\mu=1}^{\ell(k)}$ are eigen values of C. Set $d_{\tilde{\varepsilon} \nu}(\theta)=\sum_{\mu=1}^{\ell(k)} \tilde{\varepsilon}_{\mu} b_{\mu}(\theta) u_{\mu \nu}(\nu=1, \cdots, \ell(k))$. Then we have

$$
A_{\alpha}\left(R_{s k}, \theta\right)=b_{s}(\theta) C b_{s}^{*}(\theta)=\sum_{\nu=1}^{\ell(k)} \lambda_{\nu}\left|d_{\nu}(\theta)\right|^{2} .
$$

Since $\operatorname{deg} b_{\nu}(\theta) \leq N(k+1)$, we have

$$
\tilde{p}\left(\left\|d_{\tilde{\varepsilon} \nu}\right\|_{\infty} \geq c_{1}(\log N(k+1))^{1 / 2}\left(\sum_{\mu=1}^{\ell(k)}\left|b_{\mu}\right|^{2}\left|u_{\mu \nu}\right|^{2}\right)^{1 / 2}\right) \leq c_{2}(N(k+1))^{-2} .
$$

Therefore we have

$$
\begin{aligned}
& \tilde{p}\left(\left\|d_{s \nu}\right\|_{\infty} \geq c_{1}(\log N(k+1))^{1 / 2}\left(\sum_{\mu=1}^{\ell(k)}\left|b_{\mu}\right|^{2}\left|u_{\mu \nu}\right|^{2}\right)^{1 / 2} \text { for some } \nu(1 \leq \nu \leq \ell(k))\right) \\
& \leq c_{2} N(k+1)^{-1} \text {. }
\end{aligned}
$$


Since

$$
\left\|A_{\alpha}\left(R_{\varepsilon k}, \cdot\right)\right\|_{\infty} \leq \sum_{\nu=1}^{\ell(k)} \lambda_{\mu}\left\|d_{\tilde{\tilde{\nu} \nu}}\right\|_{\infty}
$$

and

$$
\begin{aligned}
\sum_{\nu=1}^{\ell(k)} \lambda_{\mu} \sum_{\mu=1}^{\ell(k)}\left|b_{\mu}\right|^{2}\left|u_{\mu \nu}\right|^{2} & =\sum_{\mu=1}^{\ell(k)}\left|b_{\mu}\right|^{2} \sum_{\mu=1}^{\ell(k)} \lambda_{\mu}\left|u_{\mu \nu}\right|^{2}=\sum_{\mu=1}^{\ell(k)}\left|b_{\mu}\right|^{2} c_{\mu \mu} \\
& =\sum_{N(k) \leq n<N(k+1)} c_{\alpha}(n, n)\left|a_{n}\right|^{2},
\end{aligned}
$$

we have

$$
\begin{aligned}
\tilde{p}\left(\sqrt{\left\|A_{\alpha}\left(R_{s k}, \cdot\right)\right\|_{\infty}} \geq\right. & \left.c_{1}(\log N(k+1))^{1 / 2}\left(\sum_{N(k) \leq n<N(k+1)} c_{\alpha}(n, n)\left|a_{n}\right|^{2}\right)^{1 / 2}\right) \\
& \leq c_{2} N(k+1)^{-1} .
\end{aligned}
$$

By the Borel-Cantelli lemma, we have

$$
\sqrt{\left\|A_{\alpha}\left(R_{s k}, \cdot\right)\right\|_{\infty}}=O\left((\log N(k+1))^{1 / 2}\left(\sum_{N(k) \leq n<N(k+1)} c_{\alpha}(n, n)\left|a_{n}\right|^{2}\right)^{1 / 2}\right) \quad \text { a.s. }(\tilde{p}) .
$$

Since

$$
\begin{aligned}
& \sum_{k=0}^{\infty}(\log N(k+1))^{1 / 2}\left(\sum_{N(k) \leq n<N(k+1)} c_{\alpha}(n, n)\left|a_{n}\right|^{2}\right)^{1 / 2} \approx \sum_{k=0}^{\infty} 2^{k / 2}\left(\sum_{2^{k} \leq j<2^{k+1}} s_{j}^{2}\right)^{1 / 2} \\
& \leq \sum_{k=0}^{\infty} 2^{k} s_{2^{k}} \leq \sum_{j=0}^{\infty} s_{j}+s_{0}<+\infty
\end{aligned}
$$

we have $\left\|A_{\alpha}\left(f_{s}, \cdot\right)\right\|_{\infty}<+\infty$ a.s. $(\tilde{p})$. We show this in the general case. Consider a random Taylor series $f_{\mathrm{s} X}(z)=\sum_{n=1}^{\infty} \varepsilon_{n} X_{n} a_{n} z^{n}$. Set

$$
T_{k}(\omega)=2^{k / 2}\left(\sum_{N(k) \leq n<N(k+1)} X_{n}(\omega)^{2} c_{\alpha}(n, n)\left|a_{n}\right|^{2}\right)^{1 / 2} .
$$

Then we have

$$
\begin{aligned}
\mathscr{E}\left[\sum_{k=0}^{\infty} T_{k}(\omega)\right] & \leq \mathscr{E}\left[\sqrt{\sum_{k=0}^{\infty} T_{k}(\omega)^{2}\left(\mathscr{E}\left[T_{k}(\omega)^{2}\right]\right)^{-1 / 2}} \sqrt{\sum_{k=0}^{\infty}\left(\mathscr{E}\left[T_{k}(\omega)^{2}\right]\right)^{1 / 2}}\right. \\
& \leq \sum_{k=0}^{\infty}\left(\mathscr{E}\left[T_{k}(\omega)^{2}\right]\right)^{1 / 2} \approx \sum_{k=0}^{\infty} 2^{k / 2}\left(\sum_{2^{k} \leq j<2^{k+1}} s_{j}^{2}\right)^{1 / 2} \\
& \leq \sum_{j=0}^{\infty} s_{j}+s_{0}<+\infty .
\end{aligned}
$$

Consequently $\sum_{k=0}^{\infty} T_{k}(\omega)<+\infty$ a.s. $(p)$. Therefore we have $\left\|A_{\alpha}\left(f_{\bullet X}, \cdot\right)\right\|_{\infty}$ $<+\infty$ a.s. $(\tilde{p})$ for each $\omega$ such that $\sum_{k=0}^{\infty} T_{k}(\omega)<+\infty$. Hence $\left\|A_{\alpha}\left(f_{X}, \cdot\right)\right\|_{\infty}$ 
$<+\infty$ a.s. $(\tilde{p} \times p)$. There exists a sequence $\stackrel{*}{\varepsilon}=\left(\varepsilon_{n}^{*}\right)_{n=1}^{\infty}$ of numbers 1 or -1 such that $\left\|A_{\alpha}\left(f_{\varepsilon}^{*}, \cdot\right)\right\|_{\infty}<+\infty$ a.s. $(p)$. For positive integers $N, \ell$ and $k$,

$$
\begin{gathered}
F_{\ell, k}^{N}=\left\{\left(x_{1}, \cdots, x_{N}\right) ; \sup _{\theta}\left|\sum_{n=1}^{N} x_{n} x_{m} a_{n} \bar{a}_{m} e^{i(n-m) \theta} c_{\alpha}\left(n, m ; 1-\frac{1}{k}\right)\right|<\ell\right\} \\
E_{\ell, k}^{N}=\left\{\omega \in \Omega ;\left(X_{1}(\omega), \cdots, X_{N}(\omega)\right) \in F_{\ell, k}^{N}\right\}
\end{gathered}
$$

and

$$
{\stackrel{*}{E_{\ell, k}^{N}}}^{N}=\left\{\omega \in \Omega ;\left(*_{\varepsilon_{1}}^{*} X_{1}(\omega), \cdots, *_{\varepsilon_{N}} X_{N}(\omega)\right) \in F_{\ell, k}^{N}\right\} .
$$

If $F_{\ell, k}^{N}$ is a cylinder set, $p\left(E_{\ell, k}^{N}\right)=p\left({ }^{*} E_{\ell, k}^{N}\right)$ (since $X_{n}^{\prime} s$ are symmetric). In the general case, using a limit process, we have $p\left(E_{\ell, k}^{N}\right)=p\left(E_{\ell, k}^{N}\right)$. Since $\lim _{\ell \rightarrow \infty} \lim _{k \rightarrow \infty} \lim _{N \rightarrow \infty} p\left(E_{\ell, k}^{N}\right)=\lim _{\ell \rightarrow \infty} \lim _{k \rightarrow \infty} \lim _{N \rightarrow \infty} p\left(E_{\ell, k}^{*}\right)=1$, we have $\left\|A_{\alpha}\left(f_{X}, \cdot\right)\right\|_{\infty}<+\infty$ a.s.. This completes the proof.

COROLlaRY 3. Let $f_{X}(z)=\sum_{n=1}^{\infty} X_{n} a_{n} z^{n}$ be a random Taylor series. Set $s_{j}=\left(\sum_{2^{j} \leq n<2 j+1} \mathscr{E}\left(X_{n}^{2}\right)\left|a_{m}\right|^{2}\right)^{1 / 2}(j=0,1, \ldots)$. If $\left(s_{j}\right)_{j=0}^{\infty}$ is a decreasing sequence and $f_{X}$ is bounded a.s., then $A_{0}\left(f_{X}, \cdot\right)$ is also bounded a.s..

Proof. It is known that if $f_{X}$ is bounded a.s., then $\sum_{j=0}^{\infty} s_{j}<+\infty$ ([1] p. 72). By Theorem 1, we have $\left\|A_{0}\left(f_{X}, \cdot\right)\right\|_{\infty}<+\infty$ a.s.. This completes the proof.

THEOREM 1'. Let $f_{X}$ and $\left(s_{j}\right)_{j=0}^{\infty}$ be the same as in Corollary 3 . If $\sum_{j=0}^{\infty} j^{1 / 2} s_{j}<+\infty$, then $V\left(f_{X}, \cdot\right)$ is bounded a.s..

Proof. First, we consider the case of Rademacher series. We denote by $Q_{s k}(z)=\sum_{2^{k} \leq n<2^{k+1}} \varepsilon_{n} n a_{n} z^{n-2^{k}}$ and $\tilde{Q}_{s k}(\theta)=Q_{e k}\left(e^{i \theta}\right)(k=0,1, \cdots)$. Since

$$
V\left(f_{\varepsilon}, \theta\right) \leq \sum_{k=0}^{\infty} \int_{0}^{1} r^{2^{k-1}}\left|Q_{\varepsilon k}(z)\right| d r \leq \sum_{k=0}^{\infty} 2^{-k}\left\|\tilde{Q}_{\varepsilon k}\right\|_{\infty},
$$

it is sufficient to show that $\sum_{k=0}^{\infty} 2^{-k}\left\|\tilde{Q}_{s}\right\|_{\infty}<+\infty$ a.s. ( $\left.\tilde{p}\right)$. By Lemma 2 , we have

$$
\tilde{p}\left(\left\|\tilde{Q}_{s k}\right\|_{\infty} \geq c_{1} k^{1 / 2}\left(\sum_{2^{k} \leq n<2^{k+1}} n^{2}\left|a_{n}\right|^{2}\right)^{1 / 2}\right) \leq c_{2} 2^{-2 k} .
$$

By the Borel-Cantelli lemma, we have 


$$
\left\|\tilde{Q}_{s k}\right\|_{\infty}=O\left(k^{1 / 2}\left(\sum_{2^{k} \leq n<2^{k+1}} n^{2}\left|a_{n}\right|^{2}\right)^{1 / 2}\right) \quad \text { a.s.. }
$$

Since

$$
\sum_{k=0}^{\infty} 2^{-k} k^{1 / 2}\left(\sum_{2^{k} \leq n<2^{k+1}} n^{2}\left|a_{n}\right|^{2}\right)^{1 / 2} \lesssim \sum_{k=0}^{\infty} k^{1 / 2} s_{k}<+\infty,
$$

we have $\sum_{k=0}^{\infty} 2^{-k}\left\|\tilde{Q}_{c k}\right\|_{\infty}<+\infty$ a.s.. In the general case, using the same method as in Theorem 1, we obtain the proof. Hence we omit the rest of the proof.

Next, we prove the following:

THEOREM 2. Let $|\alpha|<1$. Let $X=\left(X_{n}\right)_{n=1}^{\infty}$ be a sequence of real valued normal Gaussian variables and $f_{X}(z)=\sum_{n=1}^{\infty} X_{n} a_{n} z^{n}$ a random Taylor series by $X$. If $\sum_{n=1}^{\infty} n^{\alpha}(\log n)\left|a_{n}\right|^{2}<+\infty$, then $A_{0}\left(f_{X}, \cdot\right)$ is bounded a.s..

LEMMA 3. Let $Y$ be a real valued Gaussian variable such that $\mathscr{E}[Y]$ $=0$ and $\mathscr{E}\left[Y^{2}\right]=\sigma$. Then for any $E \in \mathfrak{B}$, we have

$$
\int_{E}|Y|^{2} d p(\omega) \leq \sigma p(E)\left(4 \log \frac{1}{p(E)}+\frac{e^{-1 / 2}}{\sqrt{\pi}}\right) .
$$

Proof. We have $s e^{-s^{2} / 4} \leq \sqrt{2} e^{-1 / 2}$. We have

$$
\begin{gathered}
\int_{E}|Y|^{2} d p(\omega)=\int_{E ;|Y|^{2} \leq \sigma 4 \log (1 / p(E))}+\int_{E ;|Y|^{2}>\sigma 4 \log (1 / p(E))}=I_{1}+I_{2}, \\
I_{1} \leq \sigma p(E) 4 \log \frac{1}{p(E)}
\end{gathered}
$$

and

$$
\begin{aligned}
I_{2} & \leq \frac{2}{\sqrt{2 \pi \sigma}} \int_{2 \sqrt{\sigma} \sqrt{\log (1 / p(E))}}^{\infty} s^{2} e^{-s^{2} / 2 \sigma} d s=\frac{\sqrt{2}}{\pi} \sigma \int_{2 \sqrt{\log (1 / p(E))}}^{\infty} s^{2} e^{-s^{2} / 2} d s \\
& \leq \frac{2}{\sqrt{\pi}} e^{-1 / 2} \sigma \int_{2 \sqrt{\log (1 / p(E))}}^{\infty} s e^{-s^{2} / 4} d s=\frac{e^{-1 / 2}}{\sqrt{\pi}} \sigma p(E) .
\end{aligned}
$$

Therefore we have

$$
\int_{E}|Y|^{2} d p(\omega) \leq \sigma p(E)\left(4 \log \frac{1}{p(E)}+\frac{e^{-1 / 2}}{\sqrt{\pi}}\right) .
$$

LEMMA 4. Set $r_{j}=1-2^{-j}$ and 


$$
A_{\alpha j}\left(f_{X}, \theta\right)=\int_{r_{j}}^{r_{j+1}}(1-r)^{-\alpha} r d r \int_{\theta-(1-r)}^{\theta+(1-r)}\left|f_{X}^{\prime}\left(r e^{i \psi}\right)\right|^{2} d \psi
$$

$j=0,1, \cdots$. Then we have, for $\theta, \varphi \in T$ such that $|\theta-\varphi|<1$.

$$
\begin{aligned}
A_{\alpha j}\left(f_{X}, \theta\right) & \leq A_{\alpha j}\left(f_{X}, \varphi\right)+2^{1+\alpha}\left(|\theta-\varphi| 2^{j \alpha}\right. \\
& \left.+\frac{1}{1-\alpha}|\theta-\varphi|^{1-\alpha}\right) \sum_{n=1}^{\infty}\left|X_{n}\right|^{2} n^{2}\left|a_{n}\right|^{2} \gamma_{j+1}^{n-1} .
\end{aligned}
$$

Proof. We can assume $0<\varphi<\theta<1$. We have

$$
\begin{aligned}
& A_{\alpha j}\left(f_{X}, \theta\right)-A_{\alpha j}\left(f_{X}, \varphi\right) \\
& =\int_{r_{j}}^{r_{j+1}}(1-r)^{-\alpha} r d r\left\{\int_{\theta-(1-r)}^{\theta+(1-r)}-\int_{\varphi-(1-r)}^{\varphi+(1-r)}\right\}\left|f_{X}^{\prime}\left(r e^{i \psi}\right)\right|^{2} d \psi \\
& =\int_{\substack{r_{j} \\
0<r<1-(\theta-\varphi) / 2}}^{r_{j+1}}(1-r)^{-\alpha} r d r\left\{\int_{\varphi-(1-r)}^{\theta+(1-r)}-\int_{\varphi-(1-r)}^{\theta-(1-r)}\right\}\left|f_{X}^{\prime}\left(r e^{i \psi}\right)\right|^{2} d \psi \\
& +\int_{\substack{r_{j} \\
1_{-(\theta-\varphi) / 2<r<1}}}^{r_{j+1}}(1-r)^{-\alpha} r d r\left\{\int_{\theta-(1-r)}^{\theta+(1-r)}-\int_{\varphi-(1-r)}^{\varphi+(1-r)}\right\}\left|f_{X}^{\prime}\left(r e^{i \psi}\right)\right|^{2} d \psi=J_{1}+J_{2}, \\
& J_{1} \leq \int_{r_{j}}^{r_{j+1}}(1-r)^{-\alpha} r d r\left\{\int_{\varphi+(1-r)}^{\theta+(1-r)}+\int_{\varphi-(1-r)}^{\theta-(1-r)}\right\}\left(\sum_{n=1}^{\infty}\left|X_{n}\right|^{2} n^{2}\left|a_{n}\right|^{2} r^{n-1} \sum_{n=1}^{\infty} r^{n-1}\right) d \psi \\
& \leq 2(\theta-\varphi) \sum_{n=1}^{\infty}\left|X_{n}\right|^{2} n^{2}\left|a_{n}\right|^{2} r_{j+1}^{n-1} \int_{r_{j}}^{r_{j+1}}(1-r)^{-1-\alpha} d r \\
& \leq 2^{1+\alpha}(\theta-\varphi) 2^{j \alpha} \sum_{n=1}^{\infty}\left|X_{n}\right|^{2} n^{2}\left|a_{n}\right|^{2} r_{j+1}^{n-1}
\end{aligned}
$$

and

$$
\begin{aligned}
J_{2} & \leq 4 \int_{\substack{r_{j} \\
1-(\theta-\varphi) / 2<r<1}}^{r_{j+1}}(1-r)^{1-\alpha} r \cdot \sum_{n=1}^{\infty}\left|X_{n}\right|^{2} n^{2}\left|a_{n}\right|^{2} r^{n-1} \cdot \sum_{n=1}^{\infty} r^{n-1} d r \\
& \leq 4 \sum_{n=1}^{\infty}\left|X_{n}\right|^{2} n^{2}\left|a_{n}\right|^{2} r_{j+1}^{n-1} \int_{1-(\theta-\varphi) / 2}^{1}(1-r)^{-\alpha} d r \\
& =\frac{2^{1+\alpha}}{1-\alpha}(\theta-\varphi)^{1-\alpha} \sum_{n=1}^{\infty}\left|X_{n}\right|^{2} n^{2}\left|a_{n}\right|^{2} r_{j+1}^{n-1} .
\end{aligned}
$$

This completes the proof.

Proof of Theorem 2. We may assume that $a_{n}$ 's are real. Since $n^{\alpha}\left|a_{n}\right|^{2}=O(1)$, we can assume that $\left|a_{n}\right| \leq n$. If $\sum_{n=1}^{\infty} n^{2}\left|a_{n}\right|^{2}<+\infty$, we have

$$
\mathscr{E}\left[\left\|A_{\alpha}\left(f_{X}, \cdot\right)\right\|_{\infty}\right] \leq \mathscr{E}\left[2 \int_{0}^{1}(1-r)^{1-\alpha} \cdot r \cdot \sum_{n=1}^{\infty}\left|X_{n}\right|^{2} n^{2}\left|a_{n}\right|^{2} r^{n-1} \cdot \sum_{n=1}^{\infty} r^{n-1} d r\right]
$$




$$
\leq \sum_{n=1}^{\infty} n^{2}\left|a_{n}\right|^{2} \cdot 2 \int_{0}^{1}(1-r)^{-\alpha} d r=\frac{2}{1-\alpha} \sum_{n=1}^{\infty} n^{2}\left|a_{n}\right|^{2}<+\infty
$$

Therefore $\left\|A_{\alpha}\left(f_{X}, \cdot\right)\right\|_{\infty}<+\infty$ a.s.. Suppose $\sum_{n=1}^{\infty} n^{2}\left|a_{n}\right|^{2}=+\infty$. We have, for each $j_{0}$,

$$
\left\|A_{\alpha}\left(f_{X}, \cdot\right)\right\|_{\infty} \leq \frac{2}{1-\alpha} \sum_{n=1}^{\infty}\left|X_{n}\right|^{2} n^{2}\left|a_{n}\right|^{2} r_{j_{0}}^{n-1}+\sum_{j=j_{0}}^{\infty}\left\|A_{\alpha j}\left(f_{X}, \cdot\right)\right\|_{\infty} \cdot
$$

Since $\sum_{n=1}^{\infty}\left|X_{n}\right|^{2} n^{2}\left|a_{n}\right|^{2} r_{j_{0}}^{n-1}<+\infty$ a.s. for each $j_{0}$, it is sufficient to show that $\sum_{j=j_{0}}^{\infty}\left\|A_{\alpha j}\left(f_{X}, \cdot\right)\right\|_{\infty}<+\infty$ a.s. for some $j_{0}$. There exists $j_{0}$ such that $\sum_{n=1}^{\infty} n^{2}\left|a_{n}\right|^{2} r_{j_{0}}^{2 n-1}>1$. For a positive integer $\ell$, let $E_{j}(\ell)$ be the event:

$$
\left\|A_{\alpha j}\left(f_{X}, \cdot\right)\right\|_{\infty} \geq \ell \log \frac{1}{1-r_{j}} \sum_{n=1}^{\infty} n^{2}\left|a_{n}\right|^{2} \int_{r_{j}}^{r_{j+1}}(1-r)^{1-\alpha} r^{2 n-1} d r .
$$

We shall show that $p\left(\lim \sup _{j \rightarrow \infty} E_{j}(\ell)\right)=0$ for some $\ell>0$. Choose a random variable $\theta_{j}(\omega)$ such that $A_{\alpha j}\left({ }_{X(\omega)}, \theta_{j}(\omega)\right)=\left\|A_{\alpha j}\left(f_{X(\omega)}, \cdot\right)\right\|_{\infty}$. Let $N$ be an integer such that $2^{N} \geq 2^{16+4|\alpha|} \max (1,1 /(1-\alpha))$. Then $2^{-(j+1)|\alpha|} \geq$ $2^{11+\alpha} \max (1,1 /(1-\alpha)) 2^{(5+|\alpha|+N) j}$ for any $j \geq 1$. Set $K=2^{j N}$ and $\psi_{k}=$ $2 \pi(k / K)(k=0,1, \cdots, K-1)$. Let $E_{j}(\ell, k)$ be the event: $E_{j}$ and $\theta_{j}(\omega)$ $\in\left(\psi_{k}-\pi / K, \psi_{k}+\pi / K\right)$. We prove $p\left(E_{j}(\ell, k)\right) \leq \exp \left(e^{-1 / 2} /(4 \sqrt{\pi})\right) 2^{-(\ell / 12) j}$ for $j \geq j_{0}$. Suppose $\omega \in E_{j}(\ell, k)$. By Lemma 4, we have

$$
\begin{aligned}
& A_{\alpha j}\left(f_{X(\omega)}, \theta_{j}(\omega)\right) \leq A_{\alpha j}\left(f_{X(\omega)}, \psi_{k}\right) \\
& \quad+2^{1+\alpha}\left(2^{(-N+\alpha) j}+\frac{1}{1-\alpha} 2^{-N(1-\alpha) j}\right) \sum_{n=1}^{\infty}\left|X_{n}(\omega)\right|^{2} n^{2}\left|a_{n}\right|^{2} r_{j+1}^{n-1} .
\end{aligned}
$$

We integrate each term by $\left.d p\right|_{E_{j(\ell, k)}}$ and use Lemma 3 . Then we have

$$
\begin{aligned}
\int_{E_{j}(\ell, k)} & A_{\alpha j}\left(f_{X(\omega)}, \theta_{j}(\omega)\right) d p(\omega) \\
\leq & \int_{E_{j}(\ell, k)} A_{\alpha j}\left(f_{X(\omega)}, \psi_{k}\right) d p(\omega)+2^{1+\alpha}\left(2^{(-N+\alpha) j}+\frac{1}{1-\alpha} 2^{-N(1-\alpha) j}\right) \\
& \times \sum_{n=1}^{\infty} n^{2}\left|a_{n}\right|^{2} r_{j+1}^{n-1} \int_{E_{j}(\ell, k)}\left|X_{n(\omega)}\right|^{2} d p(\omega)=I_{1}+I_{2}, \\
I_{1}= & \int_{r_{j}}^{r_{j+1}}(1-r)^{-\alpha} r d r \int_{\psi_{k}-(1-r)}^{\psi_{k+(1-r)}} d \psi\left\{\int_{E_{j}(\ell, k)}\left|\sum_{n=1}^{\infty} X_{n} n a_{n} r^{n-1} \cos (n-1) \psi\right|^{2} d p(\omega)\right. \\
& \left.\quad+\int_{E_{j}(\ell, k)}\left|\sum_{n=1}^{\infty} X_{n} n a_{n} r^{n-1} \sin (n-1) \psi\right|^{2} d p(\omega)\right\} \\
\leq & 2 \int_{r_{j}}^{r_{j+1}}(1-r)^{1-\alpha} r \cdot \sum_{n=1}^{\infty} n^{2}\left|a_{n}\right|^{2} r^{2 n-2} d r p\left(E_{j}(\ell, k)\right)
\end{aligned}
$$




$$
\times\left(4 \log \frac{1}{p\left(E_{j}(\ell, k)\right)}+\frac{e^{-1 / 2}}{\sqrt{\pi}}\right),
$$

and

$$
\begin{aligned}
& I_{2} \leq 2^{1+\alpha} \max \left(1, \frac{1}{1-\alpha}\right) 2^{(|\alpha|-N) j}\left(\sum_{n=1}^{\infty} n^{4} r_{j+1}^{n-1}\right) p\left(E_{j}(\ell, k)\right) \\
& \times\left(4 \log \frac{1}{p\left(E_{j}(\ell, k)\right)}+\frac{e^{-1 / 2}}{\sqrt{\pi}}\right) \\
& \leq 2^{11+\alpha} \max \left(1, \frac{1}{1-\alpha}\right) 2^{(5+|\alpha|-N) j} p\left(E_{j}(\ell, k)\right) \times\left(4 \log \frac{1}{p\left(E_{j}(\ell, k)\right)}+\frac{e^{-1 / 2}}{\sqrt{\pi}}\right) \\
&\left(\text { since } \sum_{n=1}^{\infty} n^{4} r_{j+1}^{n-1} \leq \sum_{n=1}^{\infty} n(n+1)(n+2)(n+3) r_{j+1}^{n-1} \leq \frac{2^{5}}{\left(1-r_{j+1}\right)^{5}}=2^{10} \cdot 2^{5 j}\right) .
\end{aligned}
$$

For $j \geq j_{0}$, we have

$$
\begin{aligned}
\int_{r_{j}}^{r_{j+1}}(1-r)^{1-\alpha} \sum_{n=1}^{\infty} n^{2}\left|a_{n}\right|^{2} r^{2 n-1} d r & \geq \int_{r_{j}}^{r_{j+1}}(1-r)^{1-\alpha} d r \geq 2^{-(j+1)|\alpha|} \\
& \geq 2^{11+\alpha} \max \left(1, \frac{1}{1-\alpha}\right) 2^{(5+|\alpha|-N) j} .
\end{aligned}
$$

Therefore we have, for $j \geq j_{0}$,

$$
\begin{aligned}
\int_{E_{j}(\ell, k)} A_{\alpha j}\left(f_{X(\omega)}, \theta_{j}(\omega)\right) d p(\omega) & \\
\leq 3 \sum_{n=1}^{\infty} n^{2}\left|a_{n}\right|^{2} \int_{r_{j}}^{r_{j+1}}(1-r)^{1-\alpha} r^{2 n-1} d r & p\left(E_{j}(\ell, k)\right) \\
& \times\left(4 \log \frac{1}{E_{j}(\ell, k)}+\frac{e^{-1 / 2}}{\sqrt{\pi}}\right) .
\end{aligned}
$$

On the other hand, we have

$$
\begin{aligned}
\int_{E_{j}(\ell, k)} & A_{\alpha j}\left(f_{X(\omega)}, \theta_{j}(\omega)\right) d p(\omega) \\
& \geq \ell p\left(E_{j}(\ell, k)\right) \log \frac{1}{1-r_{j}} \sum_{n=1}^{\infty} n^{2}\left|a_{n}\right|^{2} \int_{r_{j}}^{r_{j+1}}(1-r)^{1-\alpha} r^{2 n-1} d r .
\end{aligned}
$$

Therefore $p\left(E_{j}(\ell, k)\right) \leq \exp \left(e^{-1 / 2} /(4 \sqrt{\pi})\right) 2^{-(\ell / 12) j}$ for $j \geq j_{0}$. Consequently, we have $p\left(E_{j}(\ell)\right) \leq \exp \left(\left(e^{-1 / 2} /(4 \sqrt{\pi})\right) 2^{(N-(\ell / 12)) j}\right.$ for $j \geq j_{0}$. Choose $\ell_{0}=12 N$ +12 . Then $p\left(E_{j}\left(\ell_{0}\right)\right) \leq \exp \left(\left(e^{-1 / 2} /(4 \sqrt{\pi})\right) 2^{-j}\right.$ for $j \geq j_{0}$. By the BorelCantelli lemma, we have $\left(\lim \sup _{j \rightarrow \infty} j>j_{0} E_{j}\left(\ell_{0}\right)\right)=0$. So we have 


$$
\begin{array}{r}
\left\|A_{\alpha}\left(f_{X}, \cdot\right)\right\|_{\infty}=O\left(\log \frac{1}{1-r_{j}} \sum_{n=1}^{\infty} n^{2}\left|a_{n}\right|^{2} \int_{r_{j}}^{r_{j+1}}(1-r)^{1-\alpha} r^{2 n-1} d r\right) \\
=O\left(\sum_{n=1}^{\infty} n^{2}\left|a_{n}\right|^{2} \int_{r_{j}}^{r_{j+1}}(1-r)^{1-\alpha} r^{2 n-1} \log \frac{1}{1-r} d r\right) \\
j=j_{0}, j_{0}+1, \ldots
\end{array}
$$

Since

$$
\begin{aligned}
& \sum_{j=0}^{\infty} \int_{r_{j}}^{r_{j+1}}(1-r)^{1-\alpha} r^{2 n-1} \log \frac{1}{1-r} d r \\
& \quad \leq \int_{0}^{1}(1-r)^{1-\alpha} r^{2 n-1} \log \frac{1}{1-r} d r \\
& \quad=\sum_{m=1}^{\infty} \frac{1}{m} \int_{0}^{1}(1-r)^{1-\alpha} r^{2 n+m-1} d r \approx \sum_{m=1}^{\infty} \frac{1}{m(n+m)^{2-\alpha}} \\
& \quad \leq \frac{1}{n^{2-\alpha}} \sum_{m=1}^{n} \frac{1}{m}+\frac{1}{n^{1-\alpha}} \sum_{m=n}^{\infty} \frac{1}{m^{2}} \approx n^{\alpha-2} \log n,
\end{aligned}
$$

we have

$$
\sum_{j=0}^{\infty} \sum_{n=1}^{\infty} n^{2}\left|a_{n}\right|^{2} \int_{r_{j}}^{r_{j+1}}(1-r)^{1-\alpha} r^{2 n-1} \log \frac{1}{1-r} d r \lesssim \sum_{n=1}^{\infty} n^{\alpha}(\log n)\left|a_{n}\right|^{2}<+\infty .
$$

Therefore $\sum_{j=j_{0}}^{\infty}\left\|A_{\alpha j}\left(f_{X}, \cdot\right)\right\|_{\infty}<+\infty$ a.s.. This completes the proof.

By Theorem 2, we can answer the converse problem to Corollary 3. That is, we can show that there exists a random Taylor series $f_{X}$ such that $\left\|f_{X}\right\|_{\infty}=+\infty$ and $\left\|A_{0}\left(f_{X}, \cdot\right)\right\|_{\infty}<+\infty$ a.s.. For example, set $a_{2^{j}}=$ $1 /(j \log j)(j=2, \cdots)$ and $a_{n}=0$ for $n \neq 2^{j}(j=2, \cdots)$. Let $X=\left(X_{n}\right)_{n=1}^{\infty}$ be the same as in Theorem 2. Then $\sum_{j=0}^{\infty}\left(\sum_{2^{j} \leq n<2^{j+1}}\left|a_{n}\right|^{2}\right)^{1 / 2}=\sum_{j=0}^{\infty} a_{2^{j}}=$ $+\infty$. Therefore $f_{X}(z)=\sum_{n=1}^{\infty} X_{n} a_{n} z^{n}$ is unbounded a.s.. On the other hand, since $\sum_{n=1}^{\infty}(\log n)\left|a_{n}\right|^{2}<+\infty$, we have $\left\|A_{0}\left(f_{X}, \cdot\right)\right\|_{\infty}<+\infty$ a.s..

The method of the proof is usual. But it has many applications. Since the case of $V\left(f_{X}, \cdot\right)$ is typical, we show some applications for $V\left(f_{X}, \cdot\right)$.

Proposition 6. Let $X=\left(X_{n}\right)_{n=1}^{\infty}$ and $f_{X}$ be the same as in Theorem 2. For any $m \geq 1$, we have with constant $c_{1}$,

$$
p\left(V\left(f_{X}, 0\right) \geq c_{1} m \int_{0}^{1} \sqrt{\sum_{n=1}^{\infty} n^{2}\left|a_{n}\right|^{2} r^{2 n-2}} d r\right) \leq e^{-m^{2}} .
$$

LEMMA 5. Let $Y$ be the same as in Lemma 3. Then for any $E \in \mathfrak{B}$, we have 


$$
\int_{E}|Y| d p(\omega) \leq \sqrt{\sigma p}(E)\left(\sqrt{2} \sqrt{\log \frac{1}{p(E)}}+\sqrt{\frac{2}{\pi}}\right) .
$$

Proof. We have

$$
\begin{aligned}
& \int_{E}|Y| d p(\omega) \leq \int_{E ;|Y| \leq \sqrt{\sigma} \sqrt{2 \log 1 /(p(E))}}+\int_{E ;|Y|>\sqrt{\sigma} \sqrt{2 \log 1 /(p(E))}} \\
& \leq \sqrt{\sigma p}(E) \sqrt{2 \log \frac{1}{p(E)}}+\frac{2}{\sqrt{2 \pi \sigma}} \int_{\sqrt{\sigma} \sqrt{2 \log 1 /(p /(E))}}^{\infty} s e^{-s^{2} / 2 \sigma} d s \\
& =\sqrt{\sigma} p(E)\left(\sqrt{2} \sqrt{\log \frac{1}{p(E)}}+\sqrt{\frac{2}{\pi}}\right) \text {. }
\end{aligned}
$$

Proof of Proposition 6. Let $E$ be the event:

$$
V\left(f_{X}, 0\right) \geq 4 \sqrt{2} m \int_{0}^{1} \sqrt{\sum_{n=1}^{\infty} n^{2}\left|a_{n}\right|^{2} r^{2 n-2}} d r .
$$

Then we have

$$
\begin{aligned}
p(E) & 4 \sqrt{2} m \int_{0}^{1} \sqrt{\sum_{n=1}^{\infty} n^{2}\left|a_{n}\right|^{2} r^{2 n-2}} d r \\
& \leq \int_{E} V\left(f_{X}, 0\right) d p(\omega) \\
& \leq 2 \int_{0}^{1} \sqrt{\sum_{n=1}^{\infty} n^{2}\left|a_{n}\right|^{2} r^{2 n-2}} d r p(E)\left(\sqrt{2} \sqrt{\log \frac{1}{p(E)}}+\sqrt{\frac{2}{\pi}}\right) .
\end{aligned}
$$

Therefore $p(E) \leq e^{-(2 m-1 / \sqrt{\pi})^{2}} \leq e^{-m^{2}}$.

Proposition 7. Under the same hypothesis of Proposition 6, for any $m<1$, we have, with constant $c_{2}$,

$$
p\left(V\left(f_{X}, 0\right) \leq c_{2} m \int_{0}^{1} \sqrt{\sum_{n=1}^{\infty} n^{2}\left|a_{n}\right|^{2} r^{2 n-2}} d r\right) \geq 1-m .
$$

LEMMA 6. Let $Y$ be the same as in Lamma 3. Then for any $E$ $\in \mathfrak{B}$, we have

$$
\int_{E}|Y| d p(\omega) \geq \sqrt{\frac{\pi}{8}} \sqrt{\sigma} p(E)^{2}
$$

Proof. Choose $a$ such that $p(|Y| \leq a)=\frac{1}{2} p(E)$. Then we have

$$
a \geq \int_{0}^{a} e^{-s^{2} / 2} d s=\sqrt{\frac{\pi}{2}} \sqrt{\sigma} p(|Y| \leq a)=\sqrt{\frac{2 \pi}{4}} \sqrt{\sigma} p(E) .
$$


Then we have

$$
\begin{aligned}
\int_{E}|Y| d p(\omega) & \geq \int_{E:|Y| \geq a}|Y| d p(\omega) \\
& \geq a p(E ;|Y| \geq a) a \frac{1}{2} p(E) \geq \frac{\sqrt{2 \pi}}{8} \sqrt{\sigma} p(E)^{2}
\end{aligned}
$$

Proof of Proposition 7. Let $E$ be the event:

$$
V\left(f_{X}, 0\right) \leq \frac{\sqrt{2 \pi}}{16} m \int_{0}^{1} \sqrt{\sum_{n=1}^{\infty} n^{2}\left|a_{n}\right|^{2} r^{2 n-2}} d r
$$

We may assume

$$
\int_{0}^{1} \sqrt{\sum_{n=1}^{\infty} n^{2}\left(\operatorname{Re} a_{n}\right)^{2} r^{2 n-2}} d r \geq \frac{1}{2} \int_{0}^{1} \sqrt{\sum_{n=1}^{\infty} n^{2}\left|a_{n}\right|^{2} r^{2 n-2}} d r .
$$

Then we have

$$
\begin{aligned}
p(E) & \frac{\sqrt{2 \pi}}{16} m \int_{0}^{1} \sqrt{\sum_{n=1}^{\infty} n^{2}\left|a_{n}\right|^{2} r^{2 n-2}} d r \\
& \geq \int_{E} V\left(f_{X}, 0\right) d p(\omega) \geq \int_{0}^{1} d r \int_{E}\left|\sum_{n=1}^{\infty} X_{n} n\left(\operatorname{Re} a_{n}\right) r^{n-1}\right| d p(\omega) \\
& \geq \frac{\sqrt{2 \pi}}{8} \int_{0}^{1} \sqrt{\sum_{n=1}^{\infty} n^{2}\left(\operatorname{Re} a_{n}\right)^{2} r^{2 n-2} \cdot p(E)^{2}} \\
& \geq \frac{\sqrt{2 \pi}}{16} \int_{0}^{1} \sqrt{\sum_{n=1}^{\infty} n^{2}\left|a_{n}\right|^{2} r^{2 n-2} d r} p(E)^{2} .
\end{aligned}
$$

Therefore we have $p(E) \leq m$. Consequently, we have

$$
p\left(V\left(f_{X}, 0\right) \geq \frac{\sqrt{2 \pi}}{16} m \int_{0}^{1} \sqrt{\sum_{n=1}^{\infty} n^{2}\left|a_{n}\right|^{2} r^{2 n-2}} d r\right) \geq 1-m .
$$

THEOREM $2^{\prime}$. Let $X=\left(X_{n}\right)_{n=1}^{\infty}$ and $f_{X}$ be the same as in Theorem 2. If

$$
\int_{0}^{1} \sqrt{\sum_{n=1}^{\infty} n^{2}\left|a_{n}\right|^{2} r^{2 n-2} \log \frac{1}{1-r} d r}<+\infty,
$$

then $\left\|V\left(f_{X}, \cdot\right)\right\|_{\infty}<+\infty$ a.s..

Proof. The proof is analogous as in Theorem 2. For the sake of completeness, we give the proof. We can assume that $a_{n}$ 's are real and $\left|a_{n}\right| \leq 1$. There is nothing to prove in the case of $\sum_{n=1}^{\infty} n^{2}\left|a_{n}\right|^{2}<+\infty$. Suppose that $\sum_{n=1}^{\infty} n^{2}\left|a_{n}\right|^{2}=+\infty$. Let $E_{j}$ be the event: 


$$
\max _{\theta} \int_{r_{j}}^{r_{j+1}}\left|f_{X}^{\prime}\left(r e^{i \theta}\right)\right| d r \geq 15 \sqrt{2} \sqrt{\log \frac{1}{1-r_{j}}} \int_{r_{j}}^{r_{j+1}} \sqrt{\sum_{n=1}^{\infty} n^{2}\left|a_{n}\right|^{2} r^{2 n-2}} d r .
$$

We shall show that $p\left(E_{j}\right) \leq \exp (1 /(3 \sqrt{\pi})) 2^{-j}$ for large $j$. Set $K=2^{4 j}$ and $\psi_{k}=2 \pi(k / K)(k=0,1, \cdots, K-1)$. Choose a random variable $\theta_{j}(\omega)$ such that

$$
\int_{r_{j}}^{r_{j+1}}\left|f_{X(\omega)}^{\prime}\left(r e^{i \theta_{j}(\omega)}\right)\right| d r=\max _{\theta} \int_{r_{j}}^{r_{j+1}}\left|f_{X(\omega)}^{\prime}\left(r e^{i \theta}\right)\right| d r .
$$

Let $E_{j}(k)(k=0, \cdots, K-1)$ be the event: $E_{j}$ and $\theta_{j}(\omega) \in\left[\psi_{k}-\pi / K\right.$, $\left.\psi_{k}+\pi / K\right)$. We prove $p\left(E_{j}(k)\right) \leq \exp (1 /(3 \sqrt{\pi})) 2^{-5 j}$ for large $j$. Suppose $\omega \in E_{j}(k)$. Then

$$
\left|f_{X(\omega)}^{\prime}\left(r e^{i \theta_{j}(\omega)}\right)\right| \leq\left|f_{X(\omega)}^{\prime}\left(r e^{i \psi k}\right)\right|+\frac{\pi}{K} \sum_{n=1}^{\infty}\left|X_{n}(\omega)\right| n^{2}\left|a_{n}\right| r^{n-2}
$$

Therefore

$$
\begin{aligned}
& \int_{r_{j}}^{r_{j+1}}\left|f_{X(\omega)}^{\prime}\left(r e^{i \theta_{j}(\omega)}\right)\right| d r \\
& \quad \leq \int_{r_{j}}^{r_{j+1}}\left|f_{X(\omega)}^{\prime}\left(r e^{i \psi_{k}}\right)\right| d r+\frac{\pi}{K} 2^{-j-1} \sum_{n=2}^{\infty}\left|X_{n}(\omega)\right| n^{2}\left|a_{n}\right| r_{j+1}^{n-2} .
\end{aligned}
$$

Integrate each term by $\left.d p\right|_{E_{j}(k)}$ and use Proposition 6. Then we have

$$
\begin{aligned}
& \int_{E_{j}(k)} d p(\omega) \int_{r_{j}}^{r_{j+1}}\left|f_{X(\omega)}^{\prime}\left(r e^{i \theta_{j}(\omega)}\right)\right| d r \\
& \leq\left(2 \int_{r_{j}}^{r_{j+1}} \sqrt{\sum_{n=1}^{\infty} n^{2}\left|a_{n}\right|^{2} r^{2 n-2}} d r+\pi 2^{-5 j-1} \sum_{n=2}^{\infty} n^{2}\left|a_{n}\right| r_{j+1}^{n-2}\right) \\
& \times p\left(E_{j}(k)\right)\left(\sqrt{2} \sqrt{\log \frac{1}{p\left(E_{j}(k)\right)}}+\sqrt{\frac{2}{\pi}}\right) .
\end{aligned}
$$

Since $\sum_{n=1}^{\infty} n^{2}\left|a_{n}\right|^{2}=+\infty$, there exists $j_{0}$ such that

$$
\int_{r_{j}}^{r_{j+1}} \sqrt{\sum_{n=1}^{\infty} n^{2}\left|a_{n}\right|^{2} r^{2 n-2}} d r \geq \pi 2^{-5 j-1} \sum_{n=2}^{\infty} n^{2}\left|a_{n}\right| r_{j+1}^{n-2}
$$

for all $j \geq j_{0}$. Then we have, for $j \geq j_{0}$

$$
\begin{aligned}
p\left(E_{j}(k)\right) & 15 \sqrt{2} \int_{r_{j}}^{r_{j+1}} \sqrt{\sum_{n=1}^{\infty} n^{2}\left|a_{n}\right|^{2} r^{2 n-2}} d r \sqrt{\log \frac{1}{1-r_{j}}} \\
\leq & 3 \int_{r_{j}}^{r_{j+1}} \sqrt{\sum_{n=1}^{\infty} n^{2}\left|a_{n}\right|^{2} r^{2 n-2}} d r p\left(E_{j}(k)\right) \\
& \times\left(\sqrt{2} \sqrt{\log \frac{1}{p\left(E_{j}(k)\right)}}+\sqrt{\frac{2}{\pi}}\right)
\end{aligned}
$$


Therefore $p\left(E_{j}(k)\right) \leq \exp (1 /(3 \sqrt{\pi})) 2^{-5 j}$ for $j \geq j_{0}$. Consequently, $p\left(E_{j}\right)$ $\leq \exp (1 /(3 \sqrt{\pi})) 2^{-j}$ for $j \geq j_{0}$. So we have

$$
\begin{aligned}
& \max _{\theta} \int_{r_{j}}^{r_{j+1}}\left|f_{X}^{\prime}\left(r e^{i \theta}\right)\right| d r=O\left(\sqrt{\log \frac{1}{1-r_{j}}} \int_{r_{j}}^{r_{j+1}} \sqrt{\sum_{n=1}^{\infty} n^{2}\left|a_{n}\right|^{2} r^{2 n-2}} d r\right) \\
&=O\left(\int_{r_{j}}^{r_{j+1}} \sqrt{\sum_{n=1}^{\infty} n^{2}\left|a_{n}\right|^{2} r^{2 n-2} \log \frac{1}{1-r}} d r\right) \\
& j=j_{0}, j_{0}+1, \ldots \text { a.s.. }
\end{aligned}
$$

Since $\int_{0}^{1} \sqrt{\sum_{n=1}^{\infty} n^{2}\left|a_{n}\right|^{2} r^{2 n-2} \log \frac{1}{1-r}} d r<+\infty$, we have

$$
\left\|V\left(f_{X}, \cdot\right)\right\|_{\infty} \leq \sum_{n=0}^{\infty}\left|X_{n}\right| n\left|a_{n}\right| r_{j_{0}}^{n-1}+\sum_{j=j_{0}}^{\infty} \max _{\theta} \int_{r_{j}}^{r_{j+1}}\left|f_{X}^{\prime}\left(r e^{i \theta}\right)\right| d r<+\infty \text { a.s.. }
$$

This completes the proof.

Next, we consider one of converse problems for Theorem 2.

THEOREM 3. Let $|\alpha|<1$ and let $f_{X}(z)=\sum_{n=1}^{\infty} X_{n} a_{n} z^{n}$ be a random Taylor series by $X=\left(X_{n}\right)_{n=1}^{\infty}$. If $\lim \sup _{N \rightarrow \infty}(\log N)^{-1} \sum_{n=1}^{N} \mathscr{E}\left[X_{n}^{2}\right] n^{\alpha}\left|a_{n}\right|^{2}=$ $+\infty$ and $n^{\alpha}\left|a_{n}\right|^{2}=O(1)$, then $\lim \sup _{N \rightarrow \infty} A_{\alpha}\left(f_{X}^{N}, \theta\right)=+\infty$ for all $\theta$ a.s..

For the proof, we use the probability space $(\tilde{\Omega} \times \Omega, \tilde{B} \times \mathfrak{B}, \tilde{p} \times p)$. We denote by $\widetilde{E}[\cdot]$ the expectation. Define a sequence $Y=\left(Y_{n}\right)_{n=1}^{\infty}$ of random variables on $\tilde{\Omega} \times \Omega$ by $Y_{n}(x, \omega)=\varepsilon_{n}(x) X_{n}(\omega)$.

LEMMA 7. Let $\left(\nu_{j}\right)_{j=0}^{\infty}\left(\nu_{0}=1\right)$ be an increasing sequence of positive integers. Set $P_{Y j}(\theta)=A_{\alpha}\left(f_{Y}^{\nu j}, \theta\right)-A_{\alpha}\left(f_{Y}^{\nu j-1}, \theta\right)$ and

$$
q_{j}=\left(\sum_{\nu j-1<n \leq \nu_{j}} \tilde{\mathscr{E}}\left(Y_{n}^{2}\right) c_{\alpha}(n, n)\left|a_{n}\right|^{2}\right)^{1 / 2} \quad(j=1,2, \cdots) .
$$

Let $E_{\mu}$ be the event:

There exists $\theta$ such that $P_{Y j}(\theta) \leq \frac{1}{4} q_{j}^{2}$ for $j=1, \cdots, \mu$.

Then we have, with positive constants $B, \beta(0<\beta<1)$,

$$
\tilde{p} \times p\left(E_{\mu}\right) \leq B \mu \nu_{\mu}^{2}\left(\sum_{j=1}^{\mu} q_{j}^{2}\right)^{1 / 2} \sup \left\{q_{j}^{-1} ; j=1, \cdots, \mu\right\} \beta^{\mu} .
$$

Proof. We denote by $\left(\Omega^{\prime}, \mathfrak{B}^{\prime}, p^{\prime}\right)=(\tilde{\Omega} \times \Omega, \widetilde{\mathfrak{B}} \times \mathfrak{B}, \tilde{p} \times p)$. Set $\Omega_{j}^{\prime}=$ $\prod_{\nu j-1<n \leq \nu j} J_{n} \times I_{n}$. The element is denoted by $\left(x_{j}, \omega_{j}\right)$. Let $\left(\Omega_{j}^{\prime}, \mathfrak{B}_{j}^{\prime}, p_{j}^{\prime}\right)$ be the usual probability space. We consider $\left(\Omega^{\prime}, \mathfrak{B}^{\prime}, p^{\prime}\right)$ as the product space $\left(\prod_{j=1}^{\infty} \Omega_{j}^{\prime}, \prod_{j=1}^{\infty} \mathfrak{B}_{j}^{\prime}, \prod_{j=1}^{\infty} p_{j}^{\prime}\right)$. Set 


$$
Q_{Y j}(\theta)=Q_{Y j}\left(x_{j}, \omega_{j}\right)(\theta)=A_{\alpha}\left(f_{Y}^{\nu j}-f_{Y}^{\nu j-1}, \theta\right)
$$

and

$$
\begin{aligned}
& R_{Y j}(\theta)=R_{Y_{j}}\left[\left(x_{1}, \omega_{1}\right), \cdots,\left(x_{j}, \omega_{j}\right)\right](\theta) \\
& =2 \operatorname{Re}\left(\sum_{\nu j-1<n \leq \nu j} Y_{n} a_{n} e^{i n \theta} \bar{\sum}_{m \leq \nu_{j}-1} Y_{m} a_{m} c_{\alpha}(n, m) e^{i m \theta}\right) .
\end{aligned}
$$

Then we have $P_{Y j}(\theta)=Q_{Y j}(\theta)+R_{Y j}(\theta)$. Let $E(\theta, j)$ be the event: $Q_{Y j}(\theta)$ $<\frac{1}{2} q_{j}^{2}$ or $R_{Y j}(\theta)<0$. We show $p^{\prime}\left(\bigcap_{j=1}^{\mu} E(\theta, j)\right) \leq \gamma^{\mu}$ for some $\gamma(0<\gamma<1)$. For any $\left\{\left(x_{k}^{*}, \omega_{k}^{*}\right)\right\}_{k=1}^{j-1}$, let $E\left[\left(x_{k}^{*}, \omega_{k}^{*}\right) ; k=1, \cdots, j-1\right](\theta)$ be the event:

$$
Q_{Y j}\left(x_{j}, \omega_{j}\right)(\theta)<\frac{1}{2} q_{j}^{2} \quad \text { or } \quad R_{Y j}\left[\left(x_{1}^{*}, \omega_{1}^{*}\right), \cdots,\left(x_{j-1}^{*}, \omega_{j-1}^{*}\right),\left(x_{j}, \omega_{j}\right)\right](\theta)<0 .
$$

By the Lemma 1, we have, with constant $\eta(0<\eta<1)$,

$$
p_{j}^{\prime}\left(Q_{Y j}(\theta) \geq \frac{1}{2} q_{j}^{2}\right) \geq \eta \text {. }
$$

Suppose $Q_{Y j}\left(\tilde{x}_{j}, \tilde{\omega}_{j}\right)(\theta) \geq \frac{1}{2} q_{j}^{2}$ and $R_{Y j}\left[\left(x_{1}^{*}, \omega_{1}^{*}\right), \cdots,\left(x_{j-1}^{*}, \omega_{j-1}^{*}\right),\left(\tilde{x}_{j}, \tilde{\omega}_{j}\right)\right](\theta)<0$ for some $\left(\tilde{x}_{j}, \tilde{\omega}_{j}\right)$. Then we have $Q_{Y j}\left(-\tilde{x}_{j}, \tilde{\omega}_{j}\right)(\theta) \geq \frac{1}{2} q_{j}^{2}$ and

$$
R_{Y j}\left[\left(x_{1}^{*}, \omega_{1}^{*}\right), \cdots,\left(x_{j-1}^{*}, \omega_{j-1}^{*}\right),\left(-\tilde{x}_{j}, \tilde{\omega}_{j}\right)\right](\theta)>0 .
$$

Therefore we have

$$
\begin{aligned}
& p_{j}^{\prime}\left(Q_{Y_{j}}(\theta) \geq \frac{1}{2} q_{j}^{2}\right. \text { and } \\
& \left.R_{Y j}\left[\left(x_{1}^{*}, \omega_{1}^{*}\right), \cdots,\left(x_{j-1}^{*}, \omega_{j-1}^{*}\right),\left(x_{j}, \omega_{j}\right)\right](\theta) \geq 0\right) \geq \frac{1}{2} \eta .
\end{aligned}
$$

That is, $p_{j}^{\prime}\left(E\left[\left(x_{k}^{*}, \omega_{k}^{*}\right) ; k=1, \cdots, j-1\right](\theta)\right) \leq 1-\frac{1}{2} \eta(=\gamma)$. We have

$$
\begin{aligned}
& p^{\prime}\left(\bigcap_{j=1}^{\mu} E(\theta, j)\right)=p_{1}^{\prime} \times \cdots \times p_{j}^{\prime}\left(\bigcap_{j=1}^{\mu} E(\theta, j)\right) \\
& \quad=\int_{\substack{\mu-1 \\
j=1}} p_{\mu}^{\prime}\left(E\left[\left(x_{k}, \omega_{k}\right) ; k=1, \cdots, \mu-1\right]\right) d\left(p_{1}^{\prime} \times \cdots \times p_{\mu-1}^{\prime}\right) \\
& \quad \leq \gamma p_{1}^{\prime} \times \cdots \times p_{\mu-1}^{\prime}\left(\bigcap_{j=1}^{\mu-1} E(\theta, j)\right) \leq \cdots \leq \gamma^{\mu} .
\end{aligned}
$$

Let $F(\theta, j)$ be the event: $P_{Y j}(\theta)<\frac{1}{2} q_{j}^{2}$. Then $F(\theta, j) \subset E(\theta, j)$. Therefore $\bigcap_{j=1}^{\mu} F(\theta, j) \subset \bigcap_{j=1}^{\mu} E(\theta, j)$. We write $\psi_{k}=2 \pi(k / K)(k=0, \cdots, K-1)$, where $K$ is an integer which will be determined later. Then we have $p^{\prime}\left(\bigcup_{k=0}^{K-1} \bigcap_{j=1}^{\mu} F\left(\psi_{k}, j\right)\right) \leq K \gamma^{\mu}$. Next, we estimate $\left\|P_{Y_{j}}^{\prime}\right\|_{\infty}$. We have

$$
\begin{aligned}
P_{Y j}(\theta)= & \sum_{\nu j-1<n \leq \nu_{j}} Y_{n} a_{n} e^{i n \theta} \sum_{m \leq \nu_{j}} Y_{m} c_{\alpha}(n, m) a_{m} e^{i m \theta} \\
& +\sum_{n \leq \nu_{j-1}} Y_{n} a_{n} e^{i n \theta} \frac{\sum_{\nu j-1<m \leq \nu_{j}} Y_{m} c_{\alpha}(n, m) a_{m} e^{i m \theta}}{}
\end{aligned}
$$


Therefore we have

$$
\begin{aligned}
\left\|P_{Y j}^{\prime}\right\|_{\infty} & \leq 4 \nu_{j} \sum_{n \leq \nu_{j}}\left|Y_{n}\right|\left|a_{n}\right| \sum_{\nu_{j-1}<m \leq \nu_{j}}\left|Y_{m}\right|\left|a_{m}\right| c_{\alpha}(n, m) \\
& \leq 4 \nu_{j} \sum_{n \leq \nu_{j}}\left|Y_{n}\right|\left|a_{n}\right| \sqrt{c_{\alpha}(n, n)} \sum_{\nu_{j-1}<m \leq \nu_{j}}\left|Y_{m}\right|\left|a_{m}\right| \sqrt{c_{\alpha}(m, m)} \\
& \leq 4 \nu_{j}^{2} \sqrt{\sum_{n \leq \nu_{j}} Y_{n}^{2}\left|a_{n}\right|^{2} c_{\alpha}(n, n)} \sqrt{\sum_{\nu_{j-1}<m \leq \nu_{j}} Y_{m}^{2}\left|a_{m}\right|^{2} c_{\alpha}(m, m)} .
\end{aligned}
$$

We have $\mathscr{E}\left[\left\|P_{Y j}^{\prime}\right\|_{\infty}\right] \leq 4 \nu_{j}^{2}\left(\sum_{k=1}^{j} q_{k}^{2}\right)^{1 / 2} q_{j}$. Consequently, we have

$$
p^{\prime}\left(\left\|P_{Y^{j}}^{\prime}\right\|_{\infty} \geq(4 \pi)^{-1} K q_{j}^{2}\right) \leq 16 \pi K^{-1} \nu_{j}^{2}\left(\sum_{k=1}^{j} q_{k}^{2}\right)^{1 / 2} q_{j}^{-1} .
$$

Let $F_{\mu}$ be the event: $\left\|P_{Y j}^{\prime}\right\|_{\infty} \leq(4 \pi)^{-1} K q_{j}^{2}$ for $j=1, \cdots, \mu$. Then

$$
p^{\prime}\left(F_{\mu}^{c}\right) \leq 16 \pi K^{-1} \mu \nu_{\mu}^{2}\left(\sum_{k=1}^{\mu} q_{k}^{2}\right)^{1 / 2} \sup \left\{q_{j}^{-1} ; j=1, \cdots, \mu\right\} .
$$

For any $\theta$, there exists $k$ such that $\left|P_{Y j}(\theta)-P_{Y j}\left(\psi_{k}\right)\right| \leq \pi K^{-1}\left\|P_{Y j}^{\prime}\right\|_{\infty}$. Therefore $P_{Y j}\left(\psi_{k}\right) \leq \pi K^{-1}\left\|P_{Y j}^{\prime}\right\|_{\infty}+P_{Y j}(\theta)$. If $(x, \omega) \in E_{\mu} \cap F_{\mu}$, then we have $\pi K^{-1}\left\|P_{Y(x, \omega) j}^{\prime}\right\|_{\infty} \leq \frac{1}{4} q_{j}^{2}$ and $P_{Y j}(\theta) \leq \frac{1}{4} q_{j}^{2}$ for some $\theta$ and $j=1, \cdots, \mu$. Therefore we have for some $k, P_{Y(x, \omega) j}\left(\psi_{k}\right) \leq \frac{1}{2} q_{j}^{2}(j=1, \cdots, \mu)$. Hence we have $E_{\mu} \cap F_{\mu} \subset \bigcup_{k=0}^{k-1} \bigcap_{j=1}^{\mu} F\left(\psi_{k}, j\right)$. That is, $E_{\mu} \subset F_{\mu}^{c} \cup \bigcup_{k=1}^{k-1} \bigcap_{j=1}^{\mu} F\left(\psi_{k}, j\right)$. Consequently, we have

$$
p^{\prime}\left(E_{\mu}\right) \leq K \gamma^{\mu}+16 \pi K^{-1} \mu \nu_{\mu}^{2}\left(\sum_{j=1}^{\mu} q_{j}^{2}\right)^{1 / 2} \sup \left\{q_{j}^{-1} ; j=1, \cdots, \mu\right\} .
$$

Let $K$ be the integer part of $\gamma^{-\mu / 2}$. Then we have, with positive constant $B$,

$$
p^{\prime}\left(E_{\mu}\right) \leq B \mu \nu_{\mu}^{2}\left(\sum_{j=1}^{\mu} q_{j}^{2}\right)^{1 / 2} \sup \left\{q_{j}^{-1} ; j=1, \cdots, \mu\right\} \gamma^{\mu / 2}
$$

This completes the proof.

Proof of Theorem 3. We can assume $\mathscr{E}\left[Y_{n}^{2}\right]=\mathscr{E}\left[X_{n}^{2}\right] \leq 1$ and $c_{\alpha}(n, n)\left|a_{n}\right|^{2} \leq 1$ for all $n$. Let $\ell(\ell \geq 2)$ be an integer. We define a sequence $\left(\nu_{j}\right)_{j=1}^{\infty}$ of integers, inductively. Set $\nu_{0}=1$. Assume that $\left\{\nu_{j}\right\}_{j=1}^{\mu-1}$ are already chosen. Then let $\nu_{\mu}$ be the smallest integer such that $\nu_{\mu}>$ $\nu_{\mu-1}$ and $\sum_{\nu \mu-1<n \leq \nu \mu} \widetilde{\mathscr{E}}\left[Y_{n}^{2}\right] c_{\alpha}(n, n)\left|a_{n}\right|^{2}\left(=q_{\mu}^{2}\right) \geq \ell$. Set $c_{\mu}=\left(\log \nu_{\mu}\right)^{-1} \sum_{k=1}^{\mu} q_{k}^{2}$. By the assumption $\lim \sup _{N \rightarrow \infty}(\log N)^{-1} \sum_{n=1}^{N} \tilde{\mathscr{E}}\left[Y_{n}^{2}\right] n^{\alpha}\left|a_{n}\right|^{2}=+\infty$ and $q_{j}^{2} \leq$ $\ell+1(j=1,2, \cdots)$, we have $\lim \sup _{\mu \rightarrow \infty} c_{\mu}=+\infty$. We have

$$
\mu \nu_{\mu}^{2}\left(\sum_{j=1}^{\mu} q_{j}^{2}\right)^{1 / 2} \sup \left\{q_{j}^{-1} ; j=1, \cdots, \mu\right\} \beta^{\mu} \leq(\ell-1)^{-1} \mu \nu_{\mu}^{3} \beta^{\mu}
$$




$$
\begin{aligned}
& =(\ell-1)^{-1} \mu \exp \left(3 \sum_{j=1}^{\mu} q_{j}^{2} \frac{1}{c_{\mu}}-\mu \log \frac{1}{\beta}\right) \\
& \leq(\ell-1)^{-1} \mu \exp \left(3(\ell+1) \frac{1}{c_{\mu}}-\log \frac{1}{\beta}\right) \mu .
\end{aligned}
$$

Since $\lim \inf _{\mu \rightarrow \infty} c_{\mu}^{-1}=0$, we have

$$
\liminf _{\mu \rightarrow \infty} \mu \nu_{\mu}^{2}\left(\sum_{j=1}^{\mu} q_{q}^{2}\right)^{1 / 2} \sup \left\{q_{j}^{-1} ; j=1, \cdots, \mu\right\} \beta^{\mu}=0 .
$$

By Lemma 7, we have $\lim \inf _{\mu \rightarrow \infty} p^{\prime}\left(E_{\mu}\right)=0$. Let $G(\ell, m)$ be the event: there exists $\theta$ such that $P_{Y j}(\theta) \leq \frac{1}{4} \ell$ for $j=m, m+1, \ldots$. Since $G(\ell, 1)$ $\subset E_{\mu}$ for all $\mu$, we have $p^{\prime}(G(\ell, 1))=0$. By the same method, we have $p^{\prime}(G(\ell, m))=0$ for all $m, \ell(m, \ell=2,3, \cdots)$. Therefore $p^{\prime}\left(\bigcup_{\ell=2}^{\infty} \bigcup_{m=1}^{\infty} G(\ell, m)\right)$ $=0$. This show that $\lim \sup _{j \rightarrow \infty} P_{Y j}(\theta)=+\infty$ holds for all $\theta$ a.s. $(\tilde{p} \times p)$. Since $A_{\alpha}\left(f_{Y}^{\nu j}, \theta\right)=P_{Y j}(\theta)+A_{\alpha}\left(f_{Y}^{\nu j-1}, \theta\right) \geq P_{Y j}(\theta)$, we have

$$
\limsup _{N \rightarrow \infty} A_{\alpha}\left(f_{Y}^{N}, \theta\right)=+\infty \quad \text { for all } \theta \text { a.s. }(\tilde{p} \times p) .
$$

There exists $\varepsilon^{*}=\left(\varepsilon_{n}^{*}\right)_{n=1}^{\infty}\left(\varepsilon_{n}^{*}=1\right.$ or -1$)$ such that $\lim \sup _{n \rightarrow \infty} A_{\alpha}\left(f_{\varepsilon^{*} X}^{*}, \theta\right)=$ $+\infty$ for all $\theta$ a.s.. Since $\left\{X_{n}\right\}_{n=1}^{\infty}$ are symmetric, (by the similar method as in Theorem 1,) we have $\lim \sup _{N \rightarrow \infty} A_{\alpha}\left(f_{X}^{N}, \theta\right)=+\infty$ for all $\theta$ a.s.. This completes the proof.

\section{REFERENCES}

[1] Kahane, J. P.; Some random series of functions, Raytheon education company, 1968.

[ 2 ] Lohwater, A. J. and Piranian, G.; On a conjecture of Lusin, Michigan Math. J., (1955), p. 63-68.

[ 3 ] Offord, A. C.; Distribution of values of random function in the unit disk, Studia Math., (1972), p. 71-106.

[ 4 ] Zygmund, A.; Trigonometric series II, Cambridge, 1959.

\section{Nagoya University}

